\title{
Exploiting EXAFS and XANES for time-resolved molecular structures in liquids
}

\author{
Christian Bressler*, I, Rafael Abela ${ }^{\mathrm{II}}$ and Majed Chergui ${ }^{\mathrm{I}}$ \\ I Ecole Polytechnique Fédérale de Lausanne (EPFL), Laboratoire de Spectroscopie Ultrarapide (LSU), BSP, 1015 Lausanne, Switzerland \\ II Swiss Light Source, Paul Scherrer Institut, 5232 Villigen-PSI, Switzerland
}

Received September 7, 2007; accepted December 19, 2007

\section{Structural dynamics / Intramolecular electron transfer / Spin crossover / \\ Femtosecond and picosecond X-ray absorption spectroscopy / Transition metal compound/ \\ Nascent radicals in solution phase}

\begin{abstract}
Time-resolved X-ray absorption fine structure (XAFS) spectroscopy with picosecond temporal resolution is a new method to observe electronic and geometric structures of short-lived reaction intermediates. It combines an intense femtosecond laser source synchronized to the X-ray pulses delivered by a synchrotron light source (SLS). We present key experiments on charge transfer reactions as well as spincrossover processes in coordination chemistry compounds next to solvation dynamics studies of photogenerated atomic radicals. These examples emphasize the observables at hand using ultrafast XAFS techniques, which include the density of states, full and even partial changes in oxidation state, and internuclear distances with milli-Angström accuracy. An outlook towards femtosecond studies and biologically relevant systems stresses the high potential of XAFS methods using new femtosecond X-ray sources like free electron lasers (XFELs).
\end{abstract}

\section{Introduction}

In photochemistry and photobiology the ultrafast redistribution of charges in the valence orbitals leads to the forming and breaking of bonds, which eventually initiates chemical activity and biological functions.

In order to deepen our understanding of the underlying elementary steps of chemical reactivity, one would ideally wish to obtain simultaneous information about both the electronic and the geometric structures of the molecule, (i) in real-time and, (ii) in naturally occurring environments. Femtosecond laser spectroscopy has matured to a powerful method to investigate the electronic structure of reacting molecules, though without providing detailed information about the positions of the nuclei during the evolving reaction, which also includes the local environment, e.g., the

\footnotetext{
* Correspondence author (e-mail: Christian.Bressler@epfl.ch)
}

surrounding solvation shell of a reacting molecule. On the other hand, static geometric structures are readily obtained exploiting X-ray tools, such as X-ray crystallography, diffraction and X-ray absorption spectroscopy. The latter has also proven its utility to obtain complementary information about the electronic structure of the X-ray absorbing element as well. Combining ultrafast laser with X-ray spectroscopies in a pump-probe scheme is thus a straightforward extension to investigate the elementary steps of chemical reactivity [1]. Over the past ten years there has been some important progress in implementing time-resolved X-ray absorption spectroscopy at synchrotrons with nanosecond and picosecond temporal resolution [2-5].

In this article we present different examples of photoexcited chemical systems in a liquid environment, probed with pulsed and tunable hard X-rays in the 3-10 keV range. The extracted information includes oxidation state changes (even fractional) and altered oscillator strengths of the central atom, over molecular symmetry changes to nearest neighbor distance elongations. This collection should therefore underline the attractive potential for exploiting time-resolved X-ray absorption spectroscopy in structural dynamics research.

\section{X-ray absorption spectroscopy}

An X-ray absorption spectrum from inner-shell electrons consists of a series of bound-bound transitions (below the ionization potential IP of the inner-shell electron), followed by the absorption edge jump itself [6]. Right above the absorption edge one observes a complicated modulation of the absorption cross section due to multiple scattering of the ionized photoelectron by its nearest neighbors in the molecule. This spectral range is also called the XANES region (X-ray absorption near-edge structure), and it contains rich information about both the electronic (below IP) and the geometric (above IP) structures including bond angles and distances.

From about $50 \mathrm{eV}$ above IP to higher energies mainly single scattering events dominate, which result in a weak oscillatory modulation of the absorption cross section. This region is called the EXAFS region (extended X-ray absorption fine structure), and delivers precise information 


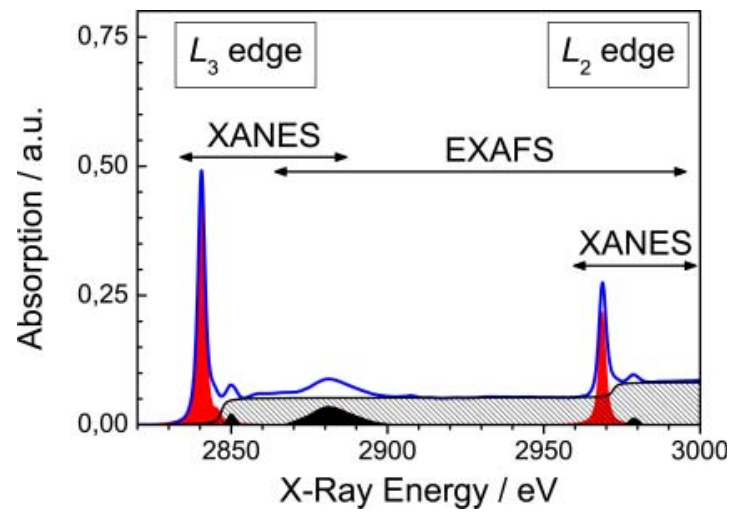

Fig. 1. X-ray absorption spectrum of $\left[\mathrm{Ru}^{\mathrm{II}}(\mathrm{bpy})_{3}\right]^{2+}$ (blue curve) around the $\mathrm{Ru} L_{3}$ and $L_{2}$ edges (stemming from from the $2 p_{3 / 2}$ and $2 p_{1 / 2}$ core levels, respectively) together with its decomposition in characteristic features: Red areas are bound-bound transitions, the shaded curve describes the bound-continuum region (whose amplitude corresponds to the so called absorption edge jump), and black areas are due to multiple-scattering resonances (near the ionization energy) and dominantly single-scattering features (at higher energies). The XANES and EXAFS regions are indictated by the arrows. Note that the EXAFS region usually extends to up to ca. $1000 \mathrm{eV}$ above the absorption edge.

about the internuclear distance of the nearest neighbors from the absorbing central atom [7]. Thus one can obtain in one single spectrum (Fig. 1) simultaneous information about the valence electronic structure next to the nuclear arrangement of the atoms in the molecular system.

The transition from multiple scattering to mainly single scattering events marks the transition from the so-called XANES to the EXAFS region, and is typically located ca. $20-50 \mathrm{eV}$ above the absorption edge, as indicated in Fig. 1. In this article we include bound-bound transitions, when we speak of XANES, and we use the expression XAFS (X-ray absorption fine structure) when we refer to either XANES and/or EXAFS.

A major advantage of XAFS is the fact that it delivers electronic and geometric structural information even in disordered systems, including solvated chromophores in liquids [1]. For coordination chemistry compounds including transition metal reaction centers in proteins, XAFS delivers unique information about the metal $d$-orbitals, which are usually optically forbidden with femtosecond laser light. Extending the pump-probe scheme towards XAFS detection thus delivers entirely new insight into the details of chemical reactivity.

In this article we present case studies of different systems, and go into the details of the different analytical treatments. These include the analysis of (i) the boundbound transitions below the ionization potential, (ii) the rather complicated XANES range with its multiple scattering resonances, and iii) the EXAFS range. One goal of this review is to link the different approaches together to a consistent picture of the underlying mechanisms in photochemistry.

\section{Experimental setup}

The basic experimental setups which we used at the Advanced Light Source (ALS) and at the Swiss Light Source

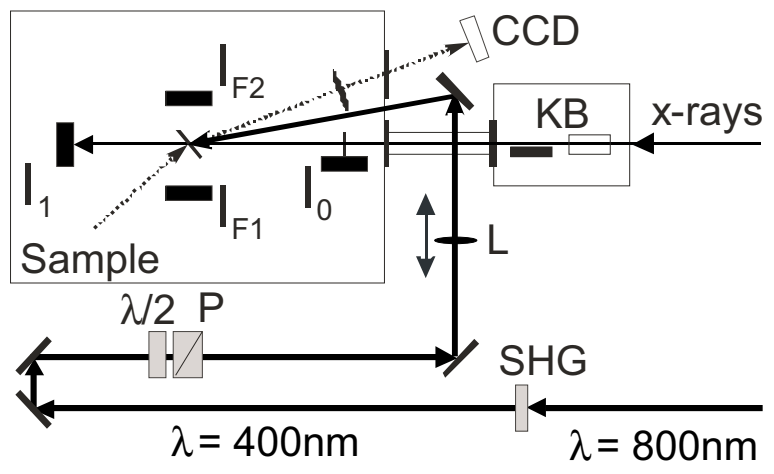

Fig. 2. Top view of the experiment with overlapping laser pump and $\mathrm{X}$-ray probe beams on the liquid sample sheet. 4 detectors $\left(I_{0}, I_{1}, I_{\mathrm{F} 1}\right.$, $I_{\mathrm{F} 2}$ ) are used for measuring the XAFS, and an imaging $\mathrm{CCD}$ detector monitors spatial overlap and beam sizes. $400 \mathrm{~nm}$ light (after frequency-doubling (SHG) the $800 \mathrm{~nm}$ fundamental beam) is adjusted for pulse energy with a $\lambda / 2$ waveplate and polarizer $(\mathrm{P})$ combination before being focused (with the lens $L$ ) onto the sample with spot sizes typically around $100-500 \mu \mathrm{m}$. The X-ray beam is focused to ca. $50 \mu \mathrm{m}$ diameter with the KB optics.

(SLS) have been described previously $[8,9,10,11]$, and will be only summarized here for the SLS setup. Briefly, Xrays from a $\mathrm{Si}(111)$ monochromator enter the experimental hutch, where they are focused to ca. $50 \mu \mathrm{m}$ diameter onto the sample with a pair of Kirkpatrick Baez (KB) mirrors (Fig. 2). X-ray signals are detected with 4 large-area avalanche photodiodes (APD), one each for transmission $\left(I_{1}\right)$ and for the incident signal scattered off a thin metal $(\mathrm{Cr})$ foil $\left(I_{0}\right)$, and via two fluorescence APDs $\left(I_{\mathrm{F} 1}, I_{\mathrm{F} 2}\right)$ for the X-ray fluorescence emitted from the sample. The sample consists of a free-flowing liquid jet (with a typical thickness in the $0.1-0.2 \mathrm{~mm}$ range), which is excited by an amplified fs laser system. Spatial overlap including a measurement of the spot sizes on the sample between both laser and X-ray beams is set via steering the laser beam onto the $\mathrm{X}$-ray spot on sample and monitored with an imaging CCD camera.

We exploit a special electron bunch filling pattern at the SLS for recording laser-pump-X-ray probe spectra (Fig. 3). The storage ring consists of 480 so called buckets, each separated by $2 \mathrm{~ns}$, in which 390 are filled (a filled bucket is also called an electron bunch) with ca. $6 \times 10^{9}$ electrons (thus generating each an average current of ca. $0.9 \mathrm{~mA}$ ), while in the subsequent $180 \mathrm{~ns}$ dark section a single densely packed (with up to 5 times more charge) hybrid electron bunch is placed. The X-ray pulse emitted from this electron bunch into the beamline (monochromatized in the $3-20 \mathrm{keV}$ range) is used to probe the photoinduced changes of the X-ray absorption of the sample.

The laser system for photoexcitation delivers $0.4 \mathrm{~mJ}$, $400 \mathrm{~nm}, 120 \mathrm{fs}$ laser pulses at $1 \mathrm{kHz}$ repetition rate, which is synchronized to the round trip frequency $(T=960 \mathrm{~ns})$, thus ensuring a constant time delay between the laser and selected probing X-ray pulses. A gated integrator delivers a sequence of output signals proportional to the input signal integrated over a fixed time window (Fig. 3). These signals are recorded with an ADC card, triggered at 4 $\mathrm{kHz}$, in order to provide a sequence of the X-ray signals when the laser is on and off, but also for recording the electronic background signal with no X-rays present. First, 


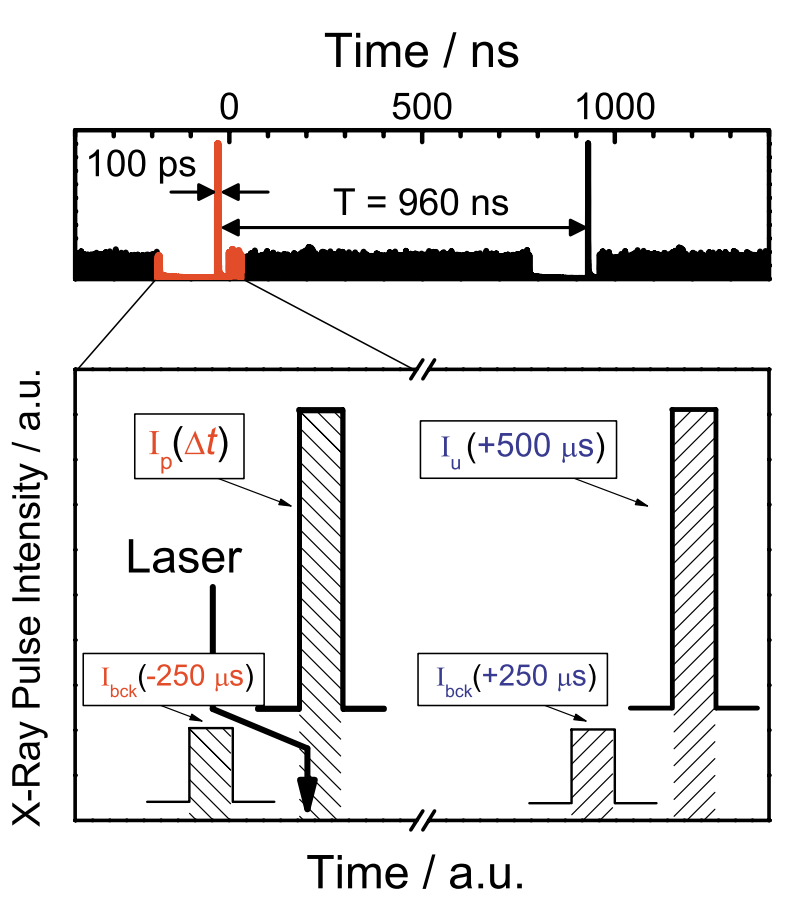

Fig. 3. Top: Bunch filling pattern at SLS (round trip time $960 \mathrm{~ns}$ ). Trains of 390 electron bunches with 2 ns spacing are followed by a $180 \mathrm{~ns}$ long empty section, in which a densely packed electron bunch (pulse width $100 \mathrm{ps}$ ) is placed $30 \mathrm{~ns}$ before the start of the bunch train. Bottom: Data acquisition scheme employed at the microXAS beamline of the Swiss Light Source. A gated integrator measures sequentially the X-ray intensity at the chosen time delay $\Delta t$, a background signal ca. $250 \mu$ s earlier and later, the X-ray signal of the unexcited sample after $500 \mu$ s (each within the indicated shaded time windows). This acquisition scheme is repeated for several thousand times per data point.

a background signal without $\mathrm{X}$-rays is recorded $\left(I_{\mathrm{bck}}(-250 \mu \mathrm{s})\right)$ ca. $250 \mu \mathrm{s}$ prior to the X-ray signal at time $\Delta t$ after laser excitation $\left(I_{p}(\Delta t)\right)$, then another background signal is recorded after ca. $250 \mu$ s, and finally, after $0.5 \mathrm{~ms}$, the X-ray signal from a fresh sample without laser excitation is recorded $\left(I_{u}(+500 \mu \mathrm{s})\right)$ [12], since the flow of the jet moves the laser-illuminated sample volume below the X-ray spot on this time scale. The pump-probe signal in transmission is defined as the transient absorption $\Delta A_{\mathrm{tr}}$ of the photoexcited sample via [1]

$$
\Delta A_{\mathrm{tr}}(E, \Delta t)=\ln \left(\frac{I_{\mathrm{unp}}}{I_{\mathrm{pum}}}\right),
$$

with $I_{\mathrm{pum}}=I_{p}(\Delta t)-I_{\mathrm{bck}}(-250 \mu \mathrm{s})$ and $I_{\mathrm{unp}}=I_{u}(+500 \mu \mathrm{s})$ $-I_{\mathrm{bck}}(+250 \mu \mathrm{s})$ being both the baseline $\left(I_{\mathrm{bck}}\right)$ corrected photoexcited and unexcited X-ray transmission signals, respectively (see Fig. 3). This strategy eliminates slow drifts on the output signal of the gated integrator next to all electronic noise sources with frequencies below a few $\mathrm{kHz}$ on the input signal (e.g., including a possible $50 \mathrm{~Hz}$ artefact from the line voltage).

The X-ray signals are measured simultaneously in transmission and fluorescence yield modes (Fig. 2), using the same data acquisition strategy for all detectors, as shown in Fig. 3. We define the transient signal $\Delta A_{\mathrm{fl}}$ in fluorescence detection mode via

$$
\Delta A_{\mathrm{fl}}(E, \Delta t)=\frac{I_{\mathrm{pum}}^{\mathrm{fl}}-I_{\mathrm{unp}}^{\mathrm{fl}}}{I_{0}}
$$

with $I_{\text {pum }}^{\mathrm{fl}}, I_{\text {unp }}^{\mathrm{fl}}$ and $I_{0}$ being each baseline corrected as described above for transmission mode. Likewise, normalization of the unpumped spectra to the incident flux yields the static XAFS of the sample.

This data acquisition scheme permits us to measure the corresponding signals for every single incident X-ray pulse, and for each data point (e.g., during a XAFS energy scan) a few thousand single X-ray pulse intensities are analyzed in order to store their averaged value and their standard deviation into the computer. We have repeatedly confirmed that this methodology delivers accuracies, which is very close to what is expected from the X-ray source flux. In other words, exploiting time-resolved XAFS at synchrotrons we are capable to measure down to the shot-noise limit of the source [8-10].

\section{Electronic structure changes}

In the following we present different case examples in time-resolved XAFS, which illustrate the rich variety of observables obtainable in a single spectrum. We focus here on electronic structure changes, which include the density of states, the oxidation state of a selected atom within the molecule, the molecular symmetry, next to its geometric structure. In the first system we emphasize the general issue of deriving an accurate excited state spectrum in a real laser-pump X-ray probe experiment. This serves as a starting ground for all the following examples.

\subsection{Photoexcited aqueous $\left[\operatorname{Ru}^{\mathrm{II}}(\mathrm{bpy})_{3}\right]^{2+}$}

Ruthenium tris $\left(2,2^{\prime}\right)$-bipyridine, $\left[\mathrm{Ru}^{\mathrm{II}}(\mathrm{bpy})_{3}\right]^{2+}$ has been widely studied due to its potential application as an efficient light-energy converter [13]. Absorption of a visible photon (e.g., $400 \mathrm{~nm}$ ) into the singlet metal-to-ligandcharge-transfer manifold ( ${ }^{1}$ MLCT) is followed by extremely fast (20 fs) intersystem crossing into the triplet MLCT states and subsequent energy relaxation within [14]. Finally, the ${ }^{3}$ MLCT state deactivates both radiatively and nonradiatively to the ground state with a measured lifetime of ca. $300 \mathrm{~ns}$ in room temperature aqueous solutions. Therefore, optical excitation leads to a change of oxidation state of the central $\mathrm{Ru}$ ion (II $\rightarrow$ III) next to a change of spin and molecular symmetry (from $D_{3}$ to $C_{2}$ ). With the current temporal resolution of $50 \mathrm{ps}$ we detected the ${ }^{3}$ MLCT state and determinded its electronic and geometric structure [15].

\subsection{Reactant X-ray absorption spectrum}

Figure 4 shows the static XAFS of the reactant (a) together with the transient absorption signal after 50 ps (b) around both the $L_{3}$ and $L_{2}$ edges. We first need to understand the ground state spectrum. The intense features $B$ at both $L_{3}$ and $L_{2}$ edges in Fig. 4 a are due to the allowed bound-bound transitions $2 p_{3 / 2,1 / 2} \rightarrow 4 d\left(t_{2 g}\right)$, respectively, into the upper empty crystal field split level of the $\mathrm{Ru}$ atom (Fig. 5). Higher-lying empty states account for the weak band (white area) shown adjacent to the $B$ features. 


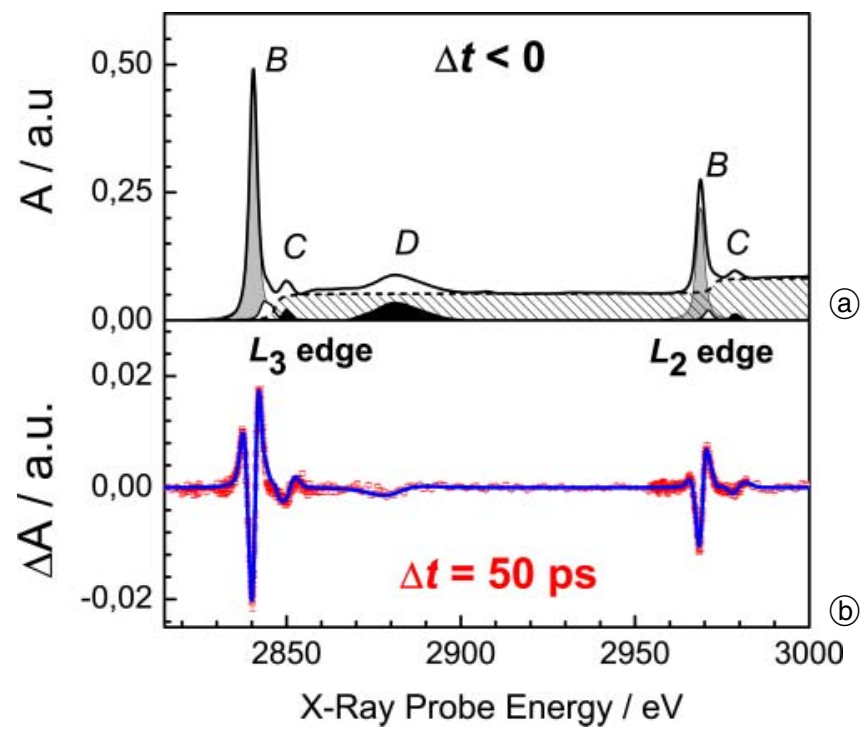

Fig. 4. (a) $L_{2,3}$ edge XAFS of aqueous $\left[\mathrm{Ru}^{\mathrm{II}}(\mathrm{bpy})_{3}\right]^{2+}$ in its ground state (thick black line) together with fits of its most prominent features (labeled), except for the two residual bands (white areas) near the ionization potential (IP). The edge step functions for IP for each state is shown as well (dashed line). (b) Transient absorption spectrum (open red circles with error bars) and a fit of this transient (blue line) using the ground state spectrum and the (energy-shifted) decomposed bands shown in a). For details see text.

Features $C$ are due to a multiple scattering resonance [16] right above the ionization potential IP (whose edge-step functions are shown in the dashed line in Fig. 4a). Finally, feature $D$ has been identified as being due to a dominantly single-scattering event, and is thus an EXAFS feature.

\subsection{Product X-ray absorption spectrum}

Figure $4 \mathrm{~b}$ shows the measured difference spectrum after $50 \mathrm{ps}$. On this time scale we have only the reactant and the relaxed ${ }^{3} \mathrm{MLCT}$ species present from the reaction

$$
\begin{aligned}
& {\left[\mathrm{Ru}^{\mathrm{II}}(\mathrm{bpy})_{3}\right]^{2+}\left({ }^{1} \mathrm{GS}\right)+h v \rightarrow} \\
& {\left[\mathrm{Ru}^{\mathrm{III}}(\mathrm{bpy})_{2} \mathrm{bpy}^{-}\right]^{2+}\left({ }^{1} \mathrm{MLCT}\right) \rightarrow\left({ }^{3} \mathrm{MLCT}\right),}
\end{aligned}
$$

since the intersystem crossing process (ISC) from singlet to the triplet MLCT state is completed after ca. 20 fs [14]. In such an experiment with only two possible states (the ground and excited states) we can calculate the transient absorption spectrum via [1]

$$
\Delta A(E, t)=f(t) \cdot[P(E)-R(E)],
$$

when both the reactant $(R(E))$ and the photoexcited product $(P(E))$ spectra are known together with the fractional population in the excited state $f$. This likewise means that we have to know $f$ very precisely in order to derive the product spectrum. This can be obtained in a separate optical measurement, but (currently) rarely during the actual pump-probe experiment. We have repeated the excitation conditions of the experiment shown in Fig. 4b, and derived a value of $f=10 \%$ [17], but the uncertainty of this value determines the accuracy in deriving the product spectrum and its interpretation. This rather simple statement affects all pump-probe type of experiments, whether optical or X-ray (diffraction or absorption), and needs to

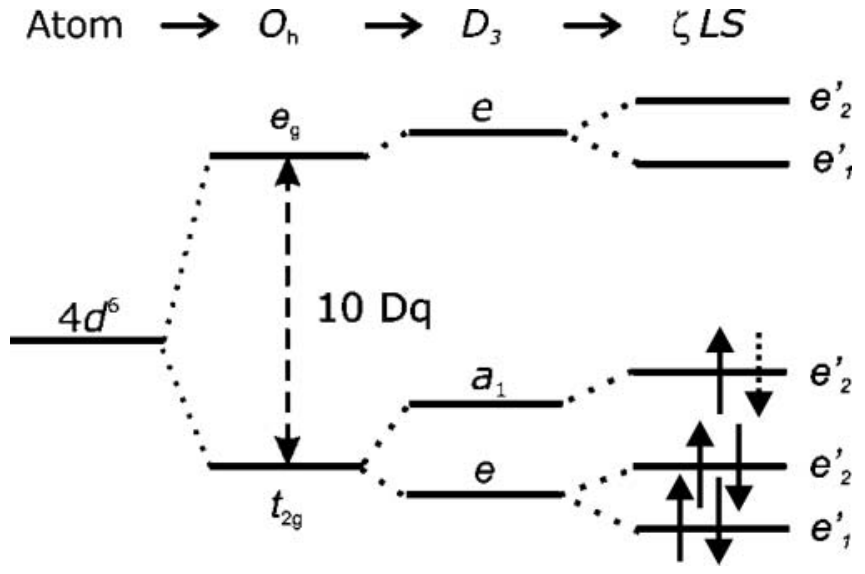

Fig. 5. Energy level scheme for a $4 d^{6}$ transition metal complex. The atomic $4 d$ orbital splits into a 6-fold degenerate $t_{2 \mathrm{~g}}$ and a 4 -fold degenerate $e_{\mathrm{g}}$ level in the octahedral field of the ligand (and separated by $10 \mathrm{Dq})$. Trigonal distortion due to the $D_{3}$ symmetry of the complex partial lifts the degeneracy, which is further lifted into 5 twofold degenerate levels by the $4 d$ spin-orbit coupling. The electron occupancy of the ground state (completely filled in $t_{2 \mathrm{~g}}$, and empty for $e_{\mathrm{g}}$ ) is indicated by the arrows, from one (dashed) is removed to the bpy ligand following photoexcitation.

be addressed precisely, before interpreting the data. Figure 6 illustrates this, where we have applied Eq. (4) to the spectra in Fig. 4a and b to derive a collection of possible candidates for the product spectrum $P(E)$.

One sees that both the spectral position(s) and intensities observed depend strongly on the fractional population used. Thus $f$ has to be well-known before commencing the interpretation. In this case, it is already evident that a new absorption feature (labeled $A$ ) is apparent in the product spectrum, which stems from the removal of one electron from the initially fully occupied $t_{2 \mathrm{~g}}$ level (dashed arrow in Fig. 5) that was promoted to the ligand system in the MLCT process. Measuring the fractional population in an independent experiment under the same conditions as the actual laser-X-ray pump-probe experiment is challenging, when its precision needs to be better than $10-20 \%$ (Fig. 6). We therefore characterized the pump beam in

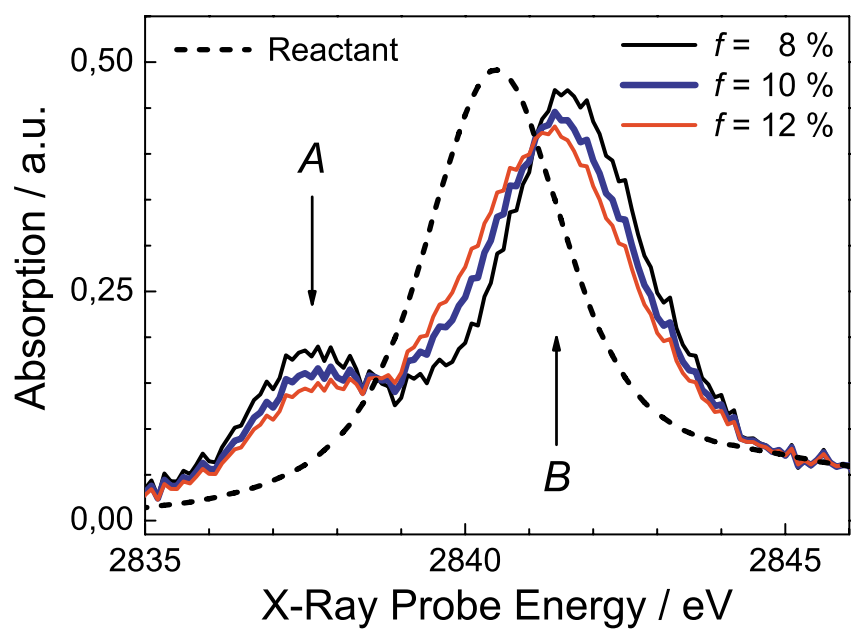

Fig. 6. Zoom into the reactant absorption spectrum (dashed line) from Fig. 4a at the $L_{3}$ edge, and product absorption spectra calculated via Eq. (4) using the indicated values for $f$. The spectral shift of feature $B$ changes hereby by nearly $0.5 \mathrm{eV}$, and the integrated areas below features $A$ and $B$ change as well. 
both experimental series as accurate as possible; nevertheless we feel only comfortable with a value of $10 \pm 1 \%$ [17]. However, spectral shifts and oscillator strengths still vary considerably within this uncertainty (see Fig. 6), but with additional knowledge of the system under investigation we can do better, as described in the next section.

\subsection{Analysis of reactant and transient spectra}

The ground state XAFS spectrum has been fitted to only the features shown in Fig. 4a, thus the edge step function (an arctan function broadened with the lifetime width of the Ru $L$ edges), features $B, C, D$, and - in a final step including contributions from higher-lying bound states [18]. The bound state absorptions $B$ (and the higher-lying bound absorptions shown in the white-area bands) were fitted using Voigt-Profiles with fixed lifetime [19, 20] $\left(1.75 \mathrm{eV}\right.$ at $L_{3}$ and $1.9 \mathrm{eV}$ at $\left.L_{2}\right)$ and monochromator widths (1.2 and $1.4 \mathrm{eV}$, respectively, for this experiment at the Advanced Light Source [21]), while features $C$ and $D$ were empirically fitted to assymmetric peak functions (Voigt profiles) with fit-optimized widths.

We have thus fitted the entire reactant XAFS in Fig. 4a to a series of peak and edge step functions

$$
R(E)=\sum_{i} g_{i}\left(E_{i}^{c}, w_{i}^{G}, w_{i}^{L}, A_{i}\right)+l_{3}\left(E, w^{L}\right)+l_{2}\left(E, w^{L}\right),
$$

with the $g_{i}$ being the aforementioned peak functions (each centered at $E_{i}^{c}$ with amplitude $A_{i}$, Gauss- and Lorentzwidths $w_{i}^{G}$ and $w_{i}^{E}$, respectively, and the edge step functions $l_{3}\left(E, w^{L}\right)$ and $l_{2}\left(E, w^{L}\right)$. Again, the Gaussian and Lorentzian widths of all bound-bound features and the Lorentzian widths of the edge step functions were kept constant during the fit [22]. These functions were then used as input functions to fit the transient absorption spectrum.

The transient signal $\Delta A$ (measured in transmission mode) in Fig. 4b is hereby given by Eq. (1), which is equal to [1]

$$
\Delta A(t)=\ln \left(\frac{I_{\text {pum }}}{I_{\text {unp }}}\right)=f(t) \cdot n_{\text {sam }} \Delta \sigma_{\text {ex }} d,
$$

linking the measured X-ray transmission intensities $I_{\text {unp }}$ and $I_{\text {pum }}$ of the dark and laser-excited sample, respectively, with the fractional population of the excited state species $f=n_{\mathrm{ex}} / n_{\text {sam }}$ and the difference between excited and ground state absorption cross sections, $\Delta \sigma_{\mathrm{ex}}=\sigma_{\mathrm{ex}}-\sigma_{\mathrm{gr}}$, in the solution phase sample with thickness $d$. The excited state absorption cross section $\sigma_{\mathrm{ex}}(E)$ is strongly related to that of the reactant cross section: from Fig. 5 we know that the oscillator strength of feature $B$ remains constant, while a new absorption feature $A$ appears after removal of one of the $t_{2 \mathrm{~g}}$ electrons in the MLCT process, which therefore appears in Fig. 6. This new feature is treated with an additional Voigt profile with the same fixed widths, as described above for the ground state XAFS. We can thus generate the excited state spectrum with the ground state functions allowing only spectral shifts for the change in oxidation state

$$
\begin{aligned}
P(E)= & \sum g_{i}\left(E_{i}^{c}+\Delta E_{i}, w_{i}^{G}, w_{i}^{L}, A_{i}\right) \\
& \left.+l_{3} E+\Delta E_{\mathrm{ox}}, w_{L}\right)+l_{2}\left(E+\Delta E_{\mathrm{ox}}, w_{L}\right),
\end{aligned}
$$

with $\Delta E_{\mathrm{Ox}}$, the energetic shift of the ionization potential, and $\Delta E_{\mathrm{val}}$, the energetic shift of the valence states, being the only adjustable parameters. The edge step is simulated by the shifted arctangent functions $l_{3}$ (for the $L_{3}$ edge) and $l_{2}\left(L_{2}\right)$ with fixed lifetime widths $w^{\mathrm{L}}$.

This treatment finally allows us to fit the raw data, i.e., the entire transient absorption spectrum in Fig. $4 \mathrm{~b}$ via

$$
\Delta A\left(f, \Delta E_{\mathrm{ox}}, \Delta E_{\mathrm{val}}\right)=f \cdot[P-R],
$$

using Eqs. (5) and (7), but with the following additional constraints: 1 . The ratio between the intensities (areas) of both the $B$ features and the edge step functions at $L_{3}$ and $L_{2}$ edges remain identical for both ground (GS) and excited (ES) states, i.e.,

$$
\left[\frac{A\left(l_{3}\right)}{A\left(l_{2}\right)}\right]_{\mathrm{GS}}=\left[\frac{A\left(l_{3}\right)}{A\left(l_{2}\right)}\right]_{\mathrm{ES}}=\left[\frac{A\left(B_{\mathrm{L} 3}\right)}{A\left(B_{\mathrm{L} 2}\right)}\right]_{\mathrm{GS}}=\left[\frac{A\left(B_{\mathrm{L} 3}\right)}{A\left(B_{\mathrm{L} 2}\right)}\right]_{\mathrm{ES}},
$$

yielding two, corresponding to the ratio of occupancies in the $2 p_{3 / 2}(=4)$ and $2 p_{1 / 2}(=2)$ initial states, and since the oscillator strength into the final $4 d\left(e_{\mathrm{g}}\right)$ state remains unchanged between ground and MLCT states (= empty).

2. The intensity ratio between the $B$ and $A$ features at the $L_{3}$ edge in the MLCT state should correspond to the electron occupancy ratio between the empty $4 d\left(e_{\mathrm{g}}\right)$ state and the single vacancy in the $4 d\left(t_{2 \mathrm{~g}}\right)$ state (Fig. 6), i.e.,

$$
\left[\frac{A\left(B_{\mathrm{L} 3}\right)}{A\left(A_{\mathrm{L} 3}\right)}\right]_{\mathrm{ES}}=4 \text {. }
$$

Respecting these relationships thus permits only the peak positions to change together with the fractional population $f$, which was kept as a fit parameter, thus we fitted Eq. (8) to only 3 parameters, (i) the fractional population $f$, (ii) the oxidation state energy shift $\Delta E_{\mathrm{ox}}$, and (iii) the shift in valence binding energy $\Delta E_{\mathrm{val}}$. The fit delivers the result shown in Fig. 4b (blue curve).

With the parameters obtained from this fit we derived the excited state spectrum (using Eq. (7)), which nicely matches the excited state data (open red circles) for $f=10.25 \%, \Delta E_{\mathrm{ox}}=+1.8 \mathrm{eV}$, and $\Delta E_{\mathrm{val}}=+0.85 \mathrm{eV}$. This agreement is also remarkable, since the fractional population was kept as a fit parameter, thus nicely confirming the laser-optical studies under (nearly) identical conditions. Figure 7 zooms into the $L_{3}$ edge region showing the reactant and product state spectra together with the observed energetic shifts (note that $\Delta E_{\mathrm{val}}$ is the difference between the IP shift and the shift of the $B$ feature, thus 1.8$0.95 \mathrm{eV}=0.85 \mathrm{eV}$.

The ionization potential for both species is depicted as lifetime-broadened step functions in the figure [18]. The energetic positions of the observed features are shown above Fig. 7. We observe a blue shift of all ground state features $B$ and $C$ next to the appearance of the new feature $A^{\prime}$ below the absorption edge $[15,18]$. The shifts are due to the photoinduced change in oxidation state of the $\mathrm{Ru}$ ion, and amount to $0.85 \mathrm{eV}$ for the valence levels $\left(=\Delta E_{\mathrm{val}}\right)$, and $1.8 \mathrm{eV}$ for the $2 p$ ionization potential (see energy level scheme in Fig. 7) [18]. The origin of the new absorption $\left(A^{\prime}\right)$ was already explained above. As a new result, we can derive the energetic splitting between the $A$ 


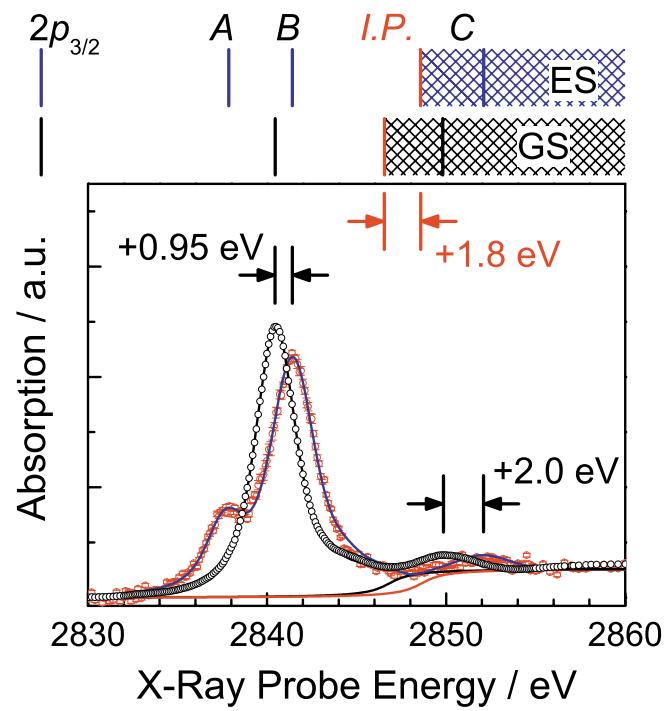

Fig. 7. Comparison of the $L_{3}$ edge XAFS of aqueous $\left[\mathrm{Ru}^{\mathrm{II}}(\mathrm{bpy})_{3}\right]^{2+}$ in its ground (thin line and points) and excited ${ }^{3}$ MLCT state (thick line and points). The edge step functions of the ionization threshold for each state is shown as well. The energy level scheme above identifies the observed features relative to the intial $2 p_{3 / 2}$ level (GS: ground state, ES: ${ }^{3}$ MLCT state).

and $B$ features, which is a measure of the crystal field energy $10 \mathrm{Dq}$ between $4 d\left(e_{\mathrm{g}}\right)$ and $4 d\left(t_{2 \mathrm{~g}}\right)$ levels (see next section). Finally, the $D$ feature (Fig. 4a) has shifted by $3 \mathrm{eV}$ (not shown here), i.e., by more than the ionization potential itself [18]. This additional shift is indicative of a slight decrease in the $\mathrm{Ru}-\mathrm{N}$ distance by ca. $30 \mathrm{pm}$, as retrieved from an analysis using both the FEFF (for the EXAFS region) and MXAN (for the XANES region) codes $[16,18]$. Details for the use of these analysis tools in time-resolved XAFS are given in Section 6.

\subsection{Density of states}

The crystal field split transitions $A$ and $B$ in the excited state can be analyzed further by calculating their oscillator strength and frequency distribution using an atomic multiplet code [23, 24]. For this purpose we have singled out their spectral signature by subtracting all the other previously determined features from the experimental spectra of the reactant and product states. This is shown in Fig. 8 at both $L_{3}$ and $L_{2}$ edges.

The stick bars in the graph represent the result of the multiplet analysis (thus displaying oscillator strengths and energy) [18], which have then been broadened with the Gaussian and Lorentzian widths due to experimental resolution and lifetime width, respectively, to generate the solid curves in the figure. While the agreement is excellent, we can also determine the crystal field splitting energy $10 \mathrm{Dq}$ for the MLCT state, which is related to the measured energetic splitting between features $A$ and $B .10 \mathrm{Dq}$ increases slightly, which results in a slight $(0.03 \AA)$ decrease in the $\mathrm{Ru}-\mathrm{N}$ bond length, when the system is in its MLCT state [18], in agreement with the above-mentioned results analyzing the EXAFS and XANES regions of the specrum $[16,18]$. An interesting observation is the fact that the $2 p_{1 / 2} \rightarrow 4 d\left(t_{2 \mathrm{~g}}\right)$ transition (thus the $A^{\prime}$ feature

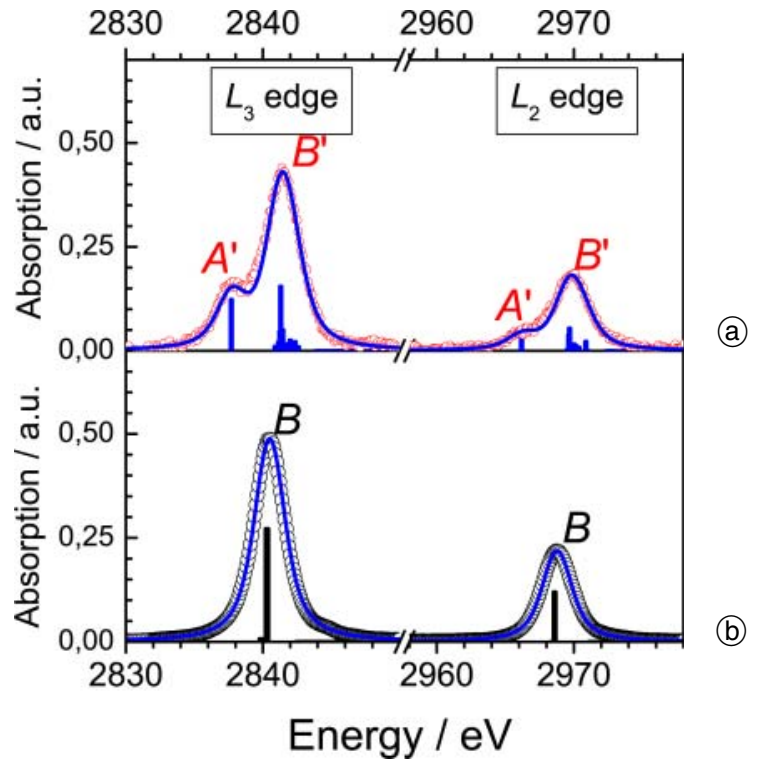

Fig. 8. Results from multiplet calculations of the $2 p_{3 / 2}\left(2 p_{1 / 2}\right) 4 d$ absorption profile together with measured data for the ground state (a) and the excited state (b) molecule (for details see Ref. [18]). The calculated transition strengths for the involved energy levels are given by the stick diagram, which - after convolution with a Lorentzian (lifetime width) and Gaussian (experimental resolution) - yield the solid curves through the data points. The experimental data was generated fom Fig. 4 after subtraction of the absorption edge and higher lying (weaker) Rydberg states. The labeled features $A$ and $B$ correspond to transitions to the $t_{2 \mathrm{~g}}$ and $e_{\mathrm{g}}$ levels, respectively (compare to Fig. 5).

at the $L_{2}$ edge in Fig. $8 \mathrm{~b}$ ) is extremely sensitive to the molecular symmetry: In an octahedral environment, it vanishes completely (spin-forbidden), while any distortion from the $O_{\mathrm{h}}$ configuration, as in the $D_{3}$ (or rather: $C_{2}$ ) symmetry of the MLCT complex, lifts the degeneracy and thus the spin selection rule [25]. In the present case, relative to the $B$ intensities, $A^{\prime}\left(L_{2}\right)$ is weaker than its (allowed) counterpart ( $A^{\prime}$ at the $L_{3}$ edge).

The example of $\left[\mathrm{Ru}^{\mathrm{II}}(\mathrm{bpy})_{3}\right]^{2+}$ demonstrates the rich variety of different observables, both electronic and structural, which can be retrieved from the XAFS. A recurring theme hereby is the knowledge of the fractional population. In principle this can be calculated beforehand using the optical cross section and the laser fluence [26, 27], but in actual transients XAFS experiments one wants to have these values measured on the fly. This is because an uncertainty in the fractional population can induce large uncertainties in the retrieval of the excited state XAFS. We got around this problem in the aforementioned case due to our additional knowledge about the system under investigation, allowing us to proceed via a complementary route. However, this is a problem of general importance to the field of structural dynamics via any observable, e.g., timeresolved electron or X-ray diffraction and crystallography. Therefore, in all these studies it has to be clearly established how the variation (uncertainty) in fractional population affects the retrieved data and, as a consequence, the interpretation.

In the next case study, we demonstrate the capability to retrieve information even about possible non-integer occupancy changes of valence levels in photoexcited systems. 


\section{Atomic radicals in solution}

The optical absorption spectrum of $\mathrm{I}^{-}$in water is characterized by two broad bands (the so-called charge-transfer-tosolvent or CTTS bands) with maxima at $225 \mathrm{~nm}$ and near $200 \mathrm{~nm}$ [28]. Excitation into these bands leads to abstraction of the electron from the negatively charged ion. The solvated electron is easily detected by its strong and broad near-IR (centred at $760 \mathrm{~nm}$ ) absorption. Its kinetics is characterised by a multiexponential decay over fs to ns, which has been well characterized in previous studies [29-31]. Important here is that the electron population corresponds to the number of photolyzed $\mathrm{I}^{-}$ions. In particular, after the initial fast processes of electron-atom recombination, nearly $1 / 5$ of the initial population of photolyzed species remains in the sample for hundreds of picoseconds [28].

We have probed the population of nascent $\mathrm{I}^{0}$ species, by X-ray absorption spectroscopy at the $L_{1}$ and $L_{3}$ edges, $50 \mathrm{ps}$, and later, after electron abstraction by the pump laser [32]. At the $L_{1}$ edge (Fig. 9a) one observes a small blue shift of the first transient peak around $5186 \mathrm{eV}$ shown in Fig. 9b with increasing delay time. Since we know that iodine radicals react towards more complex products on a diffusion-governed time scale, we have to take these processes into account, and the following reactions also occur on the pico- to nanosecond time scales following photoionization:

$$
\begin{aligned}
& \mathrm{I}^{-}+h v \rightarrow \mathrm{I}^{0}+\mathrm{e}^{-} \\
& \mathrm{I}^{0}+\mathrm{I}^{-} \rightarrow \mathrm{I}_{2}^{-} \\
& \mathrm{I}_{2}^{-}+\mathrm{I}_{2}^{-} \rightarrow \mathrm{I}_{3}^{-}+\mathrm{I}^{-}
\end{aligned}
$$

amongst other reaction channels. Therefore, in order to analyze the transient XAFS, Eq. (4) has to be modified to include these processes via

$$
\Delta A(E, t)=\sum f_{i}(t)\left[P_{i}(E)-R_{i}(E)\right],
$$

which requires both the knowledge of the participating product spectrum and its fractional (and time-dependent) population $f_{\mathrm{i}}(t)$. This is not a trivial task; while the different product populations can in principle be inferred from optical studies, the spectra of both $\mathrm{I}^{0}$ and $\mathrm{I}_{2}^{-}$have never been recorded before (while those of the reactant $\mathrm{I}^{-}$and the product $\mathrm{I}_{3}^{-}$are known or have been measured by us $[28,35])$.

We are currently in the process of analyzing this sequence quantitatively taking all reaction products into account. However, as a starting point we can already exploit the fact that at the $50 \mathrm{mM}$ concentration used in Fig. 9, the $\mathrm{I}^{0}$ radicals should be the dominant product species after 50 ps (to $>90 \%$ ), so that we can use Eq. (4) again. Still, it is not easy to know the fractional population with much precision, since laser excitation was performed via two-photon absorption of $400 \mathrm{~nm}$ light. Currently, we feel that values between around $20 \%$ seem most realistic, but we have calculated different product spectra using values of $f$ in the $10-30 \%$ range.

Figure 10 compiles the results from this analysis. The $\mathrm{I}^{-}$XAS is characterised by an edge around $5.2 \mathrm{keV}$, followed by marked modulations at higher energies [28] (not

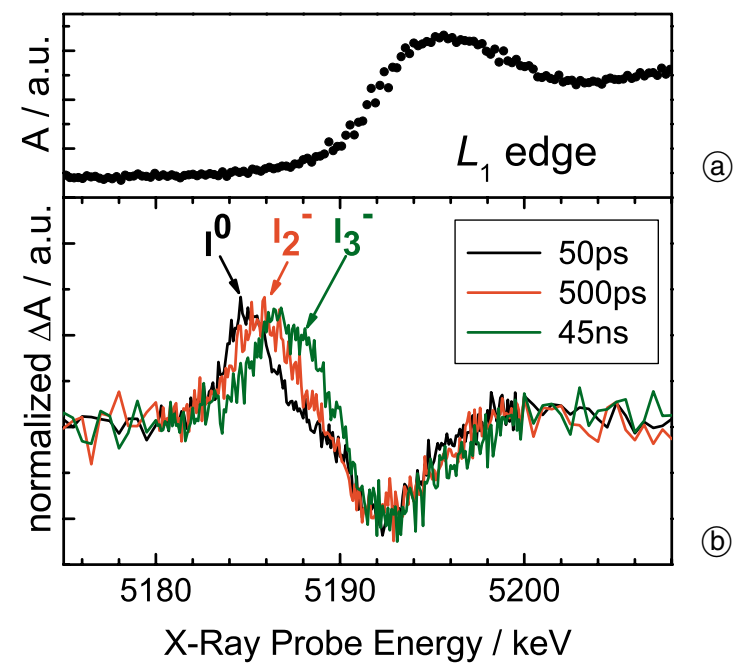

Fig. 9. (a) Static X-ray absorption spectrum of aqueous iodide. (b) Transient absorption spectra of photoexcited iodide after $50 \mathrm{ps}$ (black), $500 \mathrm{ps}$ (red), and $45 \mathrm{~ns}$ (green). The actual amplitudes have been scaled to the same height as indicated by the arrows.

shown here for $\left.L_{1}\right)$. Being isoelectronic with $\mathrm{Xe}, \mathrm{I}^{-}$does not show bound-bound core transitions at the $L_{1}$-edge, and the modulations in the XANES region are all due to above-ionization threshold multiple scattering of the photoelectron on the solvent species [33]. Removal of the electron from $\mathrm{I}^{-}$should lead to formation of $\mathrm{I}^{0}$ with a hole in the $5 p$ shell, that can then be accessed by excitation of the $2 s$ core orbital [28]. Therefore, the appearance of the $2 s \rightarrow 5 p$ resonance is a fingerprint of $\mathrm{I}^{0}$ formation, and the (shifted) spectrum of (solid) $\mathrm{I}_{2}$ has been added to compare the relative intensities.

Values of $f<10 \%$ are unreasonable, since the absorption around $5190 \mathrm{eV}$ decreases to below the pre edge value. For $f$ around $20 \%$ range we observe an oxidation state shift of ca. $2 \mathrm{eV}$, which is within the expected range according to our studies on photoexcited $\left[\mathrm{Ru}^{\mathrm{II}}(\mathrm{bpy})_{3}\right]^{2+}$ (see Section 4.1.3). In both cases a valence electron with an orbital node at the nucleus has been removed, while larger blue shifts occur for the removal of a valence $s$ elec-

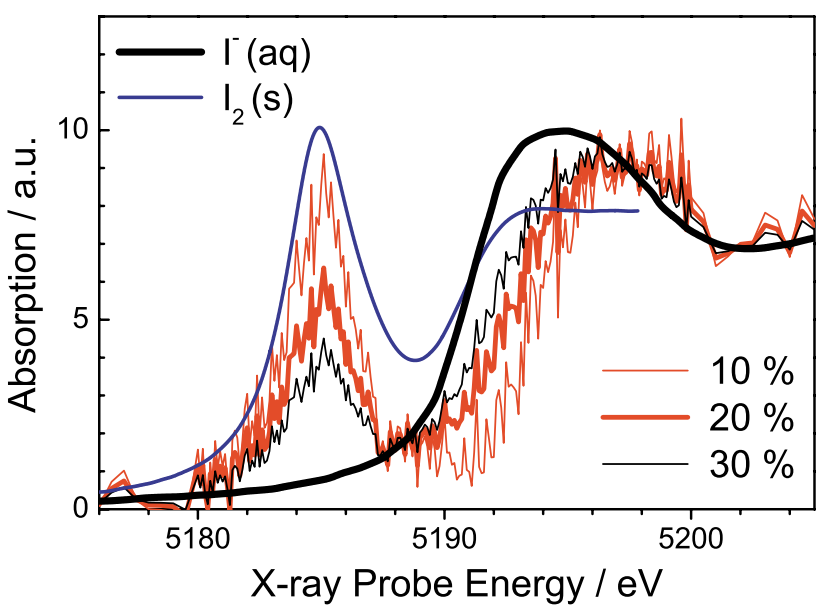

Fig. 10. Static X-ray absorption spectrum of aqueous iodide $(50 \mathrm{mM})$, solid iodine (redshifted in energy), and calculated product spectra using Eq. (4) with the indicated fractional populations $f$. From laser-optical studies we assume an $f$ in the $10-20 \%$ range to be the correct value for nascent iodine atoms. 


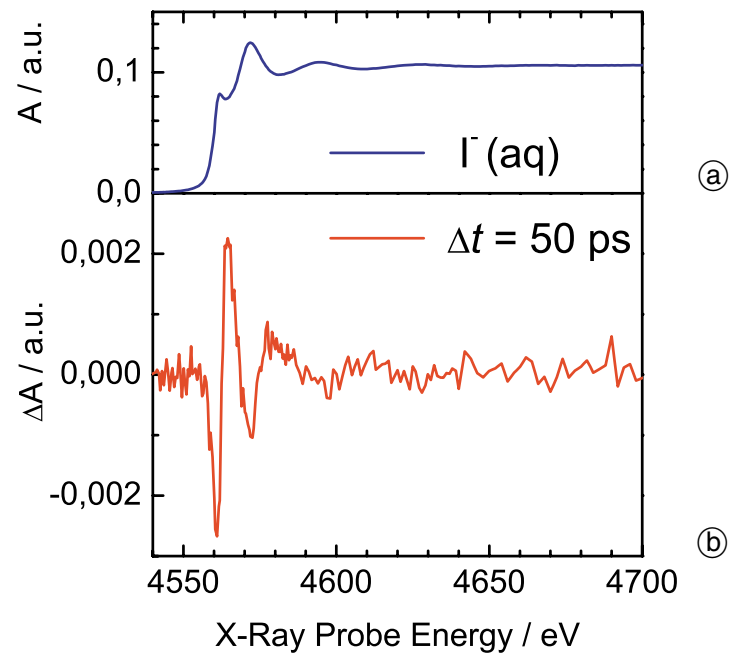

Fig. 11. (a) $L_{3}$-edge absorption spectra of aqueous $\mathrm{I}^{-}(0.1 \mathrm{M})$, (b) difference absorption spectrum $(\Delta A) 50$ ps after laser excitation.

tron (up to $3 \mathrm{eV}$, resulting from an $8 \mathrm{eV}$ increase in the $1 s$ binding energy, which is partially compensated by a $4.9 \mathrm{eV}$ increase in the valence binding energy [34]). While we are still analyzing these findings [35], it appears from Fig. 10 that the atomic $2 s \rightarrow 5 p$ transition has a decreased oscillator strength compared to the quasi isoelectronic $\mathrm{I}_{2}$. Since XANES probes the occupancy of valence orbitals, this may suggest a partial refilling of the $5 p$ orbital via electron back transfer from the caging solvent molecules, maybe concomitant with the solvent reorganization we observe in the above edge regions. Figure 11 shows that the transient spectral changes continue to within the EXAFS region, which hints towards a dramatic change in the local structure around the photoexcited reactant. [28] This spectrum, however, was measured in a $0.1 \mathrm{M}$ solution, and after 50 ps we expect a mixture of iodide, iodine atoms, diiodide and triiodide to be present in the solution (via Eqs. (11)-(13), which should also modify the transient XAFS. $50 \mathrm{mM}$ spectra around the $L_{3}$ edge will help us in interpreting the spectral changes observed around this edge [35], similar to the case of $L_{1}$ XAFS described above.

Nevertheless, the above results represent the first example of the observation of the changes in the solvent shell structure after light-induced electronic structure changes in an atomic solute, which cannot be retrieved by optical techniques.

\section{Geometric structure changes}

While the above examples focused on the electronic structure of the transient chemical species, we also discussed the retrieval of local structural information from the analysis of the XANES region. In the following we will concentrate on the local geometric structural changes, which can be extracted from both the XANES and the EXAFS regions. Next to demonstrating the accuracy of such measurements, these studies also prepare the grounds for the analysis of femtosecond XAFS changes (see Section 7), including coherent motion within the photoexcited mole- cule. When dealing with non-equilibrium dynamics processes, one can no longer rely on the prior knowledge of equilibrated structures, thus the analysis needs to be able to exclude a multitude of possible transient structures, when descending into femtosecond structural dynamics research.

\subsection{Spin crossover at room temperature}

Iron(II)-tris(2,2')-bipyridine, $\left[\mathrm{Fe}^{\mathrm{II}}(\mathrm{bpy})_{3}\right]^{2+}$ represents a typical example of a low spin (LS) compound, which can undergo light-induced spin crossover to a quintet state [36, 37]. Its characteristic energy level scheme is shown in Fig. 12. The absorption spectrum of $\left[\mathrm{Fe}^{\mathrm{II}}(\mathrm{bpy})_{3}\right]^{2+}$ is characterized by an intense broad band centred at $520 \mathrm{~nm}$ due to the singlet Metal-to-Ligand-Charge-Transfer ( ${ }^{1}$ MLCT) state. Photoexcitation into this band (or to higher energies) is followed by a cascade of intersystem crossing steps through singlet, triplet and quintet MLCT and ligand-field (LF) states, which brings the system to the lowest-lying (HS) quintet state, ${ }^{5} \mathrm{~T}_{2}$, with almost unit quantum yield in $<1$ ps [38, 39]. This state relaxes non-radiatively to the LS ground state within $\sim 0.6 \mathrm{~ns}$ in aqueous solutions at room temperature.

Figure 13a shows the static XAFS spectrum of the first $200 \mathrm{eV}$ above the Fe K absorption edge of a $25 \mathrm{mM}$ aqueous solution of $\left[\mathrm{Fe}^{\mathrm{II}}(\mathrm{bpy})_{3}\right]^{2+}$. It is characterized by a number of XANES features (labeled A to D), that have already been discussed for similar Fe-compounds [40, 41]. The features from ca. $50 \mathrm{eV}$ above the edge are all single scattering features, in particular the $E$-feature. The exact assignment of the XANES features will not be discussed here, but all have been shown to undergo significant changes upon SCO [40-43]. This is also the case here, as can be seen in Fig. 13b, which shows the transient difference spectrum measured 50 ps after excitation. In particular, the $B / C$ features gain intensity, while shifting to lower energy (inset in Fig. 13a). The D-band intensity decreases, and the E-feature shifts to lower energies. Most of these changes are attributed to the metal-ligand bond changes. Additional changes in the high energy region (see Section 6.3) are also observed, which clearly point to a signif-

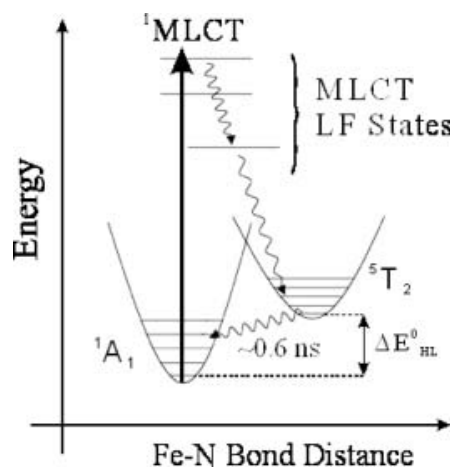

Fig. 12. Energy-level scheme of $\left[\mathrm{Fe}^{\mathrm{II}}(\mathrm{bpy})_{3}\right]^{2+}$ with its photochemical cycle [37]. A laser pulse excites the system into its metal-to-ligand charge transfer (MLCT) state, from which it cascades over MLCT and metal-centered ligand field (LF) states into the high spin (HS) ${ }^{5} \mathrm{~T}_{2}$ state. In room temperature aqueous solutions it relaxes nonradiatively back to the ground state within $0.6 \mathrm{~ns}[39,44]$. 


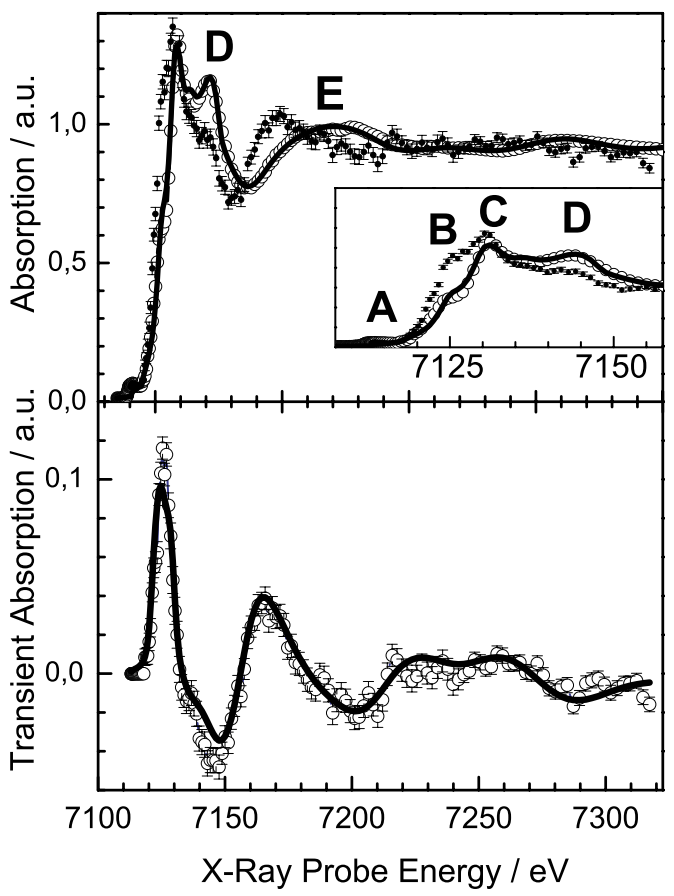

Fig. 13. (a) LS ground state XAFS spectrum of $\left[\mathrm{Fe}(\mathrm{bpy})_{3}\right]^{2+}$ together with its fit curve (solid line, the inset zooms into the edge region) (b) Transient XAFS after $50 \mathrm{ps}$ and its fit curve (solid line).

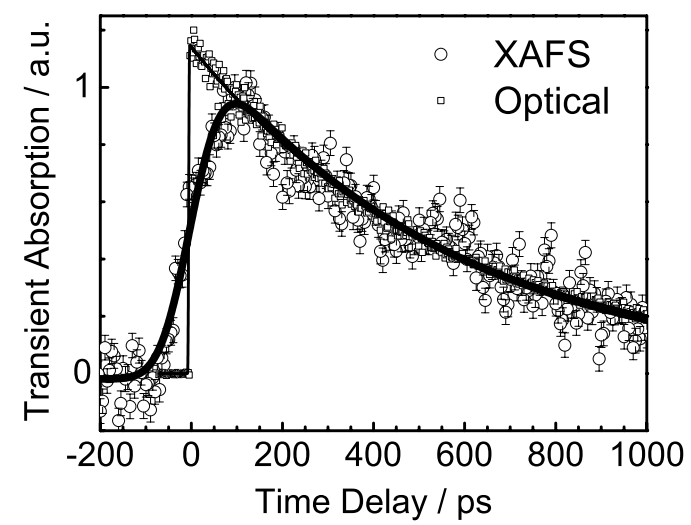

Fig. 14. Kinetics of the transient XAFS signal of aqueous $\left[\mathrm{Fe}^{\mathrm{II}}(\mathrm{bpy})_{3}\right]^{2+}$ at room temperature (open circles), recorded at $7126 \mathrm{eV}$ (at the $B$ feature), and that of the optical signal (open squares) recorded in transmission at $523 \mathrm{~nm}$, which reflects the repopulation of the ground state. The solid lines represent monoexponential fit curves, which in the case of the X-ray signal has been convoluted with the $70 \mathrm{ps}$ width of the X-ray pulse.

icant Fe-N bond change. That these changes are only due to the HS complex is seen in Fig. 14, which compares the time evolution of the absorption changes at the $B$ feature with the kinetics of ground state recovery measured by optical pump-probe spectroscopy [39]. The latter reflects the repopulation of the LS state by the decay of the HS state and it perfectly matches the X-ray data.

\subsection{EXAFS analysis of the reactant and product spectra}

The (static) extended X-ray absorption fine structure (EXAFS) is a measure of the local environment around an absorbing atom. As a function of the photoelectron wave- vector $k$ it can be described via the so-called EXAFS equation [6]

$$
\begin{aligned}
\chi(k)= & \sum_{j} \frac{S_{0}^{2} N_{j} f_{j}(k)}{k R_{j}^{2}} \cdot \mathrm{e}^{-2 R_{j} / \lambda(k)} \cdot \mathrm{e}^{-2 k^{2} \sigma_{j}^{2}} \\
& \times \sin \left[2 k R_{j}+\delta_{j}(k)\right]
\end{aligned}
$$

with the index $j$ classifying shells of identical backscatterers, each accompanied by their coordination number $N_{j}$, backscattering amplitude $f_{j}$, distance $R_{j}$ with a mean square fluctuation of $\sigma_{j}^{2}$ (Debye-Waller factor), and $\delta_{j}$ describing the $k$-dependent central atom phase shift of the absorber (decreasing roughly linear with increasing $k$ for all elements). Finally, $S_{0}^{2}$ and $\lambda(k)$ are the amplitude reduction factor and the mean free path of the photoelectron wave, respectively.

We fitted the $k^{3}$-weighted EXAFS spectra of the LS complex [5, 44] using the scattering amplitudes and phases obtained with the FEFF8.20 code [45, 46]. Hereby only the $\mathrm{Fe}-\mathrm{N}$ bond distances $R(\mathrm{Fe}-\mathrm{N}$ ) (and their DebyeWaller factors $\sigma^{2}(\mathrm{~N})$ ) were freely optimized in the fit (restrained to all $6 \mathrm{~N}$-atoms having the same values), while we started off with the published $\left[\mathrm{Fe}^{\mathrm{II}}(\mathrm{bpy})_{3}\right]^{2+}$ structure from crystallographic measurements [47]. The parameters obtained from the ground state fit are $S_{0}^{2}=0.75$, $\sigma^{2}(\mathrm{~N})=0.006 \AA^{2}$, and finally $R_{\mathrm{LS}}(\mathrm{Fe}-\mathrm{N})=1.99(0.02) \AA$ [44], which agrees nicely with the crystallographically deterimed value of 1.967 (0.006) $\AA$ [47].

With these values we fitted the high spin spectrum, which was generated via Eq. (4) using $f=22 \%$ [48], and keeping the above-mentioned parameters (apart from $R(\mathrm{Fe}-\mathrm{N}))$ constant. Hereby we noticed a considerable improvement of the quality of the fit, when we included a spectral shift $\Delta E$ in the fit procedure, which optimized at a value around $-2.5 \mathrm{eV}$. The resulting fit obtained for the high spin state $R_{\mathrm{HS}}(\mathrm{Fe}-\mathrm{N})=2.19(0.04) \AA$ [5, 44]. This delivers a result for the $\mathrm{Fe}-\mathrm{N}$ bond elongation of 0.2 (0.04) $\AA$ [44], and the accuracy depends strongly on the knowledge of the input parameters, including the ionization potential of the high spin complex. Figure 15 shows a comparison of the Fourier-transformed experimental and fitted spectra (not corrected for the central atom phase shift, which corresponds to a ca. $0.5 \AA$ horizontal displacement of the curves shown in Fig. 15) used in this analysis.

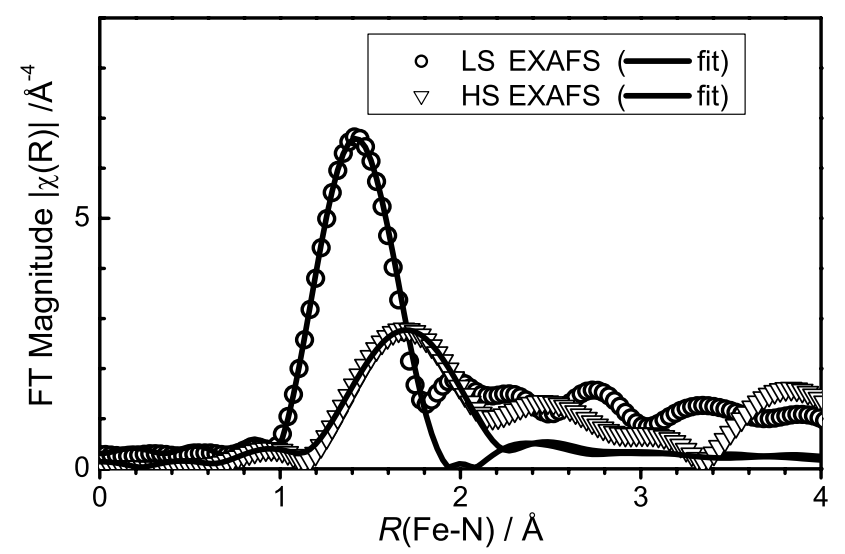

Fig. 15. Fourier transformed EXAFS spectra for the low spin (LS) and high spin (HS) states of $\left[\mathrm{Fe}^{\mathrm{II}}(\mathrm{bpy})_{3}\right]^{2+}$ (data points) and the fitted spectra limited to the first shell only (solid curves). 
In another approach we have fitted the XANES region of the ground state spectrum (Fig. 13) with the MXAN code $[49,50]$, which uses the so called full multiple scattering (FMS) approach, avoiding any a priori selection of the relevant MS paths, and the Muffin-Tin (MT) approximation for the shape of the atomic potentials [51], which are recalculated and optimized at each step of the leastsquare minimization procedure. With the so determined ground state parameters we used a recent extension of the MXAN code [16] to directly fit the difference absorption spectrum in Fig. 13b (solid line), which yields $\Delta R(\mathrm{Fe}-\mathrm{N})=0.19(0.03) \AA$, thus with a slightly better accuracy than the previous analysis of the EXAFS range.

Although both approaches (EXAFS via FEFF, and XANES via MXAN) for the HS structure deliver identical results (within their uncertainties) and are also in line with theoretical predictions [36], we might be able to achieve more information from the data. This is not so important for the present case, but is vital for our understanding of what we can actually extract from time-resolved XAFS experiments in the future. It is also important in view of the interpretation of femtosecond experiments (see Section 7). Since transition state geometric structures are currently completely unknown, femtosecond XAFS offers the attractive potential to measure just these with high precision. In preparation of these next steps in time-resolved XAFS, we have embarked into an advanced analysis of the transient XAFS spectrum of photoexcited $\left[\mathrm{Fe}^{\mathrm{II}}(\text { bpy })_{3}\right]^{2+}$.

\subsection{Analysis of the transient EXAFS}

For analyzing the transient spectrum we are tempted to apply equation 4 to derive the transient EXAFS spectrum (using Eq. (15)) via

$$
\begin{aligned}
& \Delta \chi\left(k, R_{j}, N_{j}, \ldots\right) \\
& =f \cdot\left[\chi_{\mathrm{ES}}\left(k, R_{j}, N_{j}, \ldots\right)-\chi_{\mathrm{GS}}\left(k, R_{j}, N_{j}, \ldots\right)\right]
\end{aligned}
$$

but now as a function of photoelectron wave vector $k$, rather than energy. In addition, we need prior knowledge of the input parameters in Eq. (15) for both the ground and excited states, which thus hampers the utility of Eq. (16) to calculate the transient spectrum. However, since most of these parameters are expected to be nearly identical for both states of the same molecule, we could adopt the strategy to first fit the ground state spectrum to derive these parameters, and then to fit only the radial distances $R_{j}$ in Eq. (16) to derive the structural changes in the excited molecule. Such a treatment would have to be performed with great care, since the $R_{j}$ 's of the bipyridine ligand cannot be independently modified. This is illustrated in Fig. 16, which shows the result of a theoretical simulation of the complex in both its LS and HS states [36]. While the $\mathrm{Fe}-\mathrm{N}$ distance changes, the bpy structure remains mainly constant (especially in the metal-centered ${ }^{5} \mathrm{~T}_{2}$ high spin state), and the $\mathrm{N}-\mathrm{Fe}-\mathrm{N}$ angle decreases by ca. $6^{\circ}$ [36]. Therefore, in the EXAFS analysis of the excited state, one has to respect the geometry of the ligand system.

In conclusion, it is not possible to use Eq. (16) in a straightforward fashion, and to exploit powerful computer

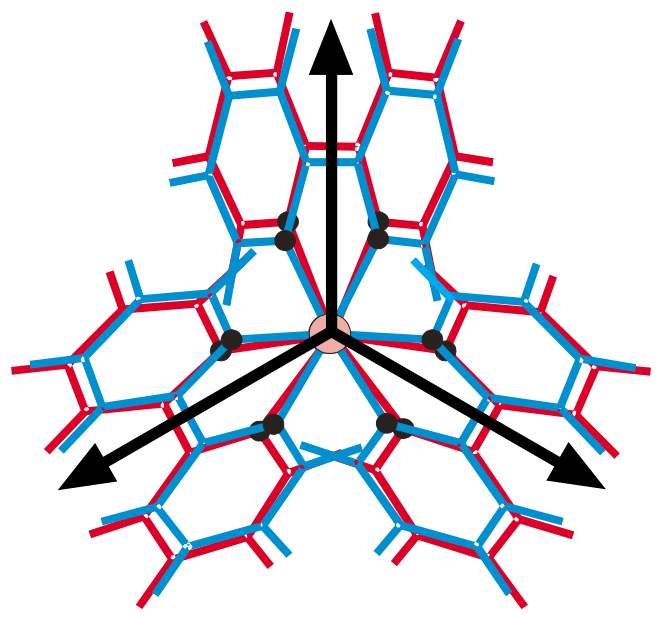

Fig. 16. Strategy for analyzing the transient EXAFS spectrum, showing the low spin (blue) and the high spin (red) structures from a DFT simulation [36]. The arrows show the direction of the bpy ligand movements, which have been used in the EXAFS analysis.

codes (like FEFF) for fitting the transient data. Also, a generated excited state spectrum (via Eqs. (4) or (16)) exhibits an increased error (by roughly $1 / f$ ). This effect is clearly seen in Fig. 10 (for iodide), for which e.g. the edge step becomes ambiguous to more than $1 \mathrm{eV}$ for fractional populations in the $10-20 \%$ range. Overall, it would be advantageous to treat only the transient spectrum (together with input provided from the well-known reactant XAFS) in a meaningful quantitative interpretation.

Finally, Eq. (16) has a major flaw: It uses the EXAFS of ground and excited states as a function of photoelectron wave vector $k$, meaning that $k=0$ corresponds to the ionization potential IP of the system. But as we have seen for the cases of iodide and $\left[\mathrm{Ru}^{\mathrm{II}}(\mathrm{bpy})_{3}\right]^{2+}$, IP changes between ground and excited states. While those shifts were related to the change in oxidation state (and thus appear not applicable in the present case with no change in oxidation state), we cannot exclude an energetic shift [52], since the rearrangement of the valence electrons may have an influence on the transition energies (and this may cause either a blue or a red shift of the spectrum). We will find below that the HS IP exhibits a red shift of ca. $1 \mathrm{eV}$, and since the experiment is performed in energy space, we have to first perform the transformation via

$$
k=\frac{2 \pi}{\hbar} \cdot \sqrt{2 m_{\mathrm{e}}(h v-I P)}
$$

(with $m_{\mathrm{e}}$ being the electron mass). Thus a fit of the transient EXAFS Eq. (16) becomes wrong, if IP differs in both states. The FEFF code offers the possibility to shift the edge energy, but in the present approach we seek to determine the edge shift from an independent analysis without involving correlated parameters.

In consequence, to overcome the above-mentioned limitations we have proceeded in the following way:

- fit the ground state EXAFS spectrum $\chi_{\mathrm{GS}}(k)$ to Eq. (15) and extract all (fit) parameters therein.

- Calculate a series of EXAFS spectra with Eq. (15) for a stepwise increase of the Fe-bpy distance (as shown in Fig. 16 for two distances) keeping all previously derived input parameters constant except for the 
respective Fe-neighbor distances $R_{j}$, which have been preset by modifying the excited state structure and respecting the bpy structure.

- Backtransform this series of EXAFS spectra into energy space via Eq. (17) using the ground state IP. This yields a collection of $\chi_{\mathrm{GS}}\left(E, I P_{\mathrm{GS}}\right)$ EXAFS spectra.

- Apply Eq. (4) to calculate transient spectra via

$\Delta \chi_{m}\left(E, \Delta E_{m}\right)=\left[P\left(E+\Delta E_{m}\right)-R(E)\right]$,

for various possible values of IP shifts $\Delta E_{m}$.

- Evaluate the least square residuals between simulation and experiment via

$R^{2}\left(i, f_{n}, \Delta E_{m}\right)=\frac{1}{(N-1)} \sum_{j=1}^{N}\left(\frac{x_{j} / f_{n}-\Delta \chi_{j, m}\left(\Delta R_{i}\right)}{\Delta x_{j} / f_{n}}\right)^{2}$,

with $x_{j}$ representing an experimental data point of the transient measurement and $\Delta x_{j}$ its measured error bar. Hereby the subscript $j$ denotes the experimental data points ( $N$ total), while $i$ refers to the Fe-bpy distance used (and the $\Delta R_{i}$ distance change with respect to the ground state, respecting the rigid bpy structure) and subscript $m$ to the utilized energy shift value $\Delta E_{m}$ between ground and excited states. Finally, $R^{2}$ is calculated for various values of the fractional population $f_{n}$. This treatment is not a fit in the usual sense. It represents merely an evaluation of the agreement between a certain model (with its utilized parameters) and the experiment. But this way we can assess the reliability of this simulation series, and also judge the limits for varying each parameter (thus determine the error for each parameter).

In Fig. 17 the static EXAFS is compared to the fitted outcome of the EXAFS calculation performed with the FEFF8.20 code over a large energy range (ca. $650 \mathrm{eV}$ above the edge). The result yields an excellent agreement with the LS structure as determined by static X-ray crystallography. The fit was done by taking into account all scattering contributions and by varying their Debye-Waller factors to best match the experimental data. In addition, we have refined the $\mathrm{Fe}-\mathrm{N}$ bond distance by setting it as a free running parameter and the obtained result of 1.97 (0.02) $\AA$ agrees very well with the published value [47].

In the next step, we have performed EXAFS calculations for several structural models with different $\mathrm{Fe}-\mathrm{N}$

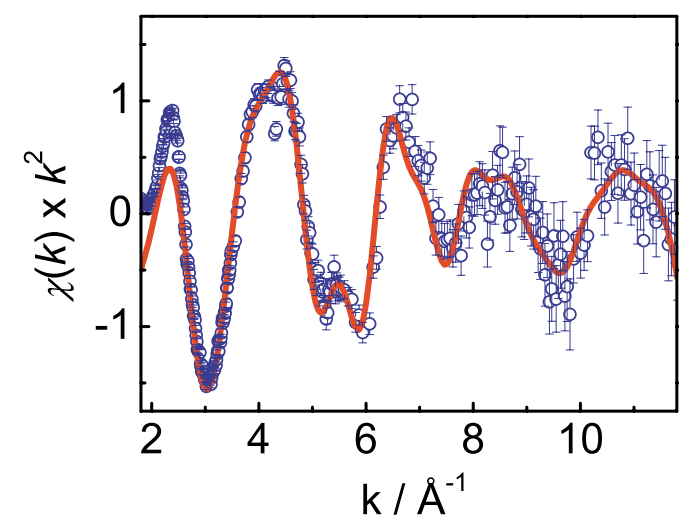

Fig. 17. LS state EXAFS spectrum together with its fit curve yielding $R_{\mathrm{Fe}-\mathrm{N}}=1.97(0.01) \AA$. distances (the actual models were obtained by moving all 3 bpy ligands symmetrically outwards from the Fe atom and thus modifying the bond lengths of all constituent atoms with respect to the absorber, see Fig. 16) in steps ranging from 0.03 to $0.005 \AA$ (in the $0-0.3 \AA$ range). This treatment also varies the $\mathrm{N}-\mathrm{Fe}-\mathrm{N}$ bond angle (decreasing angle for increasing $\mathrm{Fe}-\mathrm{N}$ distance). We then calculated the transient EXAFS for various values of $f$ from 10 to $30 \%$ (in steps of $1 \%$ ), using the ground state fit curve from Fig. 17 and evaluated the agreement with the experiment via Eq. (19). The result of these calculations is shown in Fig. 18 for the best value of the chemical shift $\Delta E_{m}=-1.2 \mathrm{eV}$, while (not shown) we have evaluated various $\Delta E_{m}$ values in the $-5-+5 \mathrm{eV}$ range.

Overall, we obtain an excellent agreement for $\Delta R(\mathrm{Fe}-\mathrm{N})=0.20(0.01) \AA$, and the resulting transient simulation is depicted in Fig. 19 together with the data. This novel approach yields uncertainties on the order of conventional state-of-the-art EXAFS studies, i.e., in the 0.01-0.001 $\AA$ range. This is because transient structural changes are rather small compared to the ground state structure (although, from the chemical point of view, $0.2 \AA$ is a rather drastic change in distance). In contrast to the fit of the ground and excited states separately, the uncertainty of the transient analysis did not increase beyond that of the ground state. In principle, the transient analysis could yield an uncertainty below that of the reactant state, since many uncertainties in the ground state EXAFS Eq. (15) cancel for the difference spectrum between two nearly identical species. With more precise knowledge of the ground state structure (e.g., from crystallographic measurements), the fit procedure for determining the excited state structural changes becomes even less ambiguous.

The above interpretation is satisfying, in that it uses the ground state structure as input, and evaluates possible excited state structures using stringent boundary conditions. In this case this means that the rigid bpy structure has been respected. This result is also identical to the one

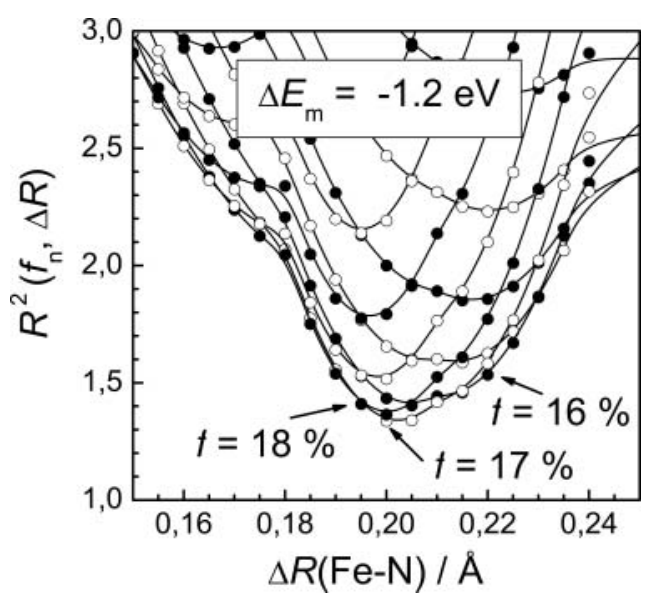

Fig. 18. Square residuals $R^{2}$ between the experimental data and transient simulations from candidate $\left[\mathrm{Fe}^{\mathrm{II}}(\mathrm{bpy})_{3}\right]^{2+}$ excited state structures, by moving simultaneously all three bpy ligands in steps of $0.005 \AA$ in the $\Delta R$ range from $0.15-0.24 \AA$. The curves serve as a guide to the eye, and connect the $R^{2}$ values for a fixed $f$-value (varied in steps of $1 \%$ from $12-28 \%$, solid and open circles display even and odd $f$ values, respectively). The transient spectra (and the square residuals shown here) have been calculated for a chemical shift value $\Delta E_{m}=-1.2 \mathrm{eV}$. 


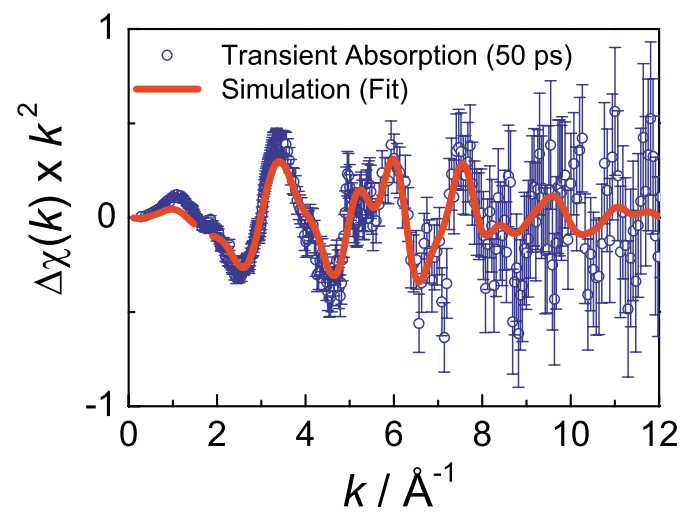

Fig. 19. Transient EXAFS after 50 ps together with the (best-fit) transient simulation for $\Delta R_{\mathrm{Fe}-\mathrm{N}}=+0.20(0.01) \AA$.

derived via the MXAN code [16]. But the latter analysis used only a rigid pirydine structure and maintained a constant $\mathrm{N}-\mathrm{Fe}-\mathrm{N}$ angle, which violates the constant bridging $\mathrm{C}-\mathrm{C}$ bond distance. Furthermore, an optimized chemical shift of $-2.8 \mathrm{eV}$ was derived in the MXAN analysis, which appears much larger than possible for a system with no change in oxidation state. This might have been a result of the utilized fractional population of $22(2) \%$, which was obtained in comparative laser-only studies [48]. But it remains not easy to reliably quantify laser-only measurements with respect to transient XAFS studies, and additional uncertainties seem unavoidable. Such an uncertainty is troublesome in view of the calculated high spin spectrum with the fitted structural parameters [53], and it is therefore becoming clear that future studies exploiting $\mathrm{X}$-ray methods should provide an in situ measurement of the involved fractional population(s). The present EXAFS analysis determined the fractional population in the fit procedure to $17(1) \%$ (Fig. 18). New calculations using the MXAN code are underway, which intend to explore the sensitivity of this code to (i) fractional populations, (ii) chemical energy shifts, and (iii) to the actual structure of the bpy ligand system, in order to clarify this issue. Indeed, a useful code in the XANES region would be mostly appreciated, since it requires only a fraction of the energy range an EXAFS analysis usually requires. This would amplify its utility towards the use of femtosecond $\mathrm{X}$-ray sources in the near and midterm (see Section 7.1), since current synchrotron-based femtosecond hard X-ray sources have extremely limited fluxes (= limited tunability during the experiment), and future ultra-intense hard X-ray sources (free electron lasers or XFELs) have technical limitations towards tunability.

\section{Perspectives for ultrafast XAFS}

\subsection{Femtosecond XAFS in chemistry}

The physical time scale for relaxation from the singlet ${ }^{1}$ MLCT to the HS state in photoexcited $\left[\mathrm{Fe}^{\mathrm{II}}(\mathrm{bpy})_{3}\right]^{2+}$ is expected to be $<1 \mathrm{ps}$, in principle occuring via several intermediate singlet and triplet states, which are each accompanied by a structural modification of the $\mathrm{Fe}-\mathrm{N}$ bond distances $[38,39]$. Optical spectroscopy has so far not been able to resolve these steps, and is unable to deter- mine their structures. Future extension of these experiments using femtosecond X-ray pulses may soon provide better insight into this relaxation process.

We have recently embarked into the field of femtosecond XAFS. For this purpose we exploited broadly tunable x-radiation extracted via the time-slicng scheme at the micro-XAS beamline at the Swiss Light Source [54]. We exploited this source to study the femtosecond events during the spin crossover process of $\left[\mathrm{Fe}^{\mathrm{II}}(\mathrm{bpy})_{3}\right]^{2+}$, monitored at the $B$ feature in Fig. 13. Figure 20a shows a preliminary result of this time-delay scan at a fixed energy.

While the analysis of this new data is still underway [55], we can already confirm that the scatter in Fig. 20a (= error bars in the figure) is entirely due to the statistical noise (shot noise) of the femtosecond X-ray source (Fig. 20b). With ca. 10 incident photons per pulse at $2 \mathrm{kHz}$ repetition rate (alternatively probing the static and photoexcited transmission intensity at $1 \mathrm{kHz}$ ) we accumulate around $10^{4}$ incident photons per second (for each the excited and dark sample) following a recent upgrade of the laser system to $2 \mathrm{kHz}$ repetition rate. We estimated in Ref. [1] the required number of X-ray photons to be $2 \times 10^{7}$ to achieve a signal to noise ratio $(S / N)$ of 1 for a hypothetical solvated iron metal compound (thus for $f=10 \%$, $c=1 \mathrm{M}$, and $\Delta \chi=1 \%$ in this case). Applying the measured values here $(f=20 \%, c=0.05 \mathrm{M}$, and $\Delta \chi=50 \%$ at the $B$ feature in Fig. 13a) then delivers ca. $5 \times 10^{5}$ required incident photons for $S / N=1$. The spectrum in Fig. 20a has $S / N \cong 5$, and we have collected $1.2 \times 10^{7}$ photons per data point on the transmission detector (both values averaged over the entire spectrum), which - after correction for the sample transmission - yields the same value, when we correct the $5 \times 10^{5}$ estimate with the measured $S / N \cong 5$ (Fig. 20). This way we show that both the source specifications as well as the sample properties match nicely, even on the femtosecond time scale, and even on a long measurement time scale (the spectrum in
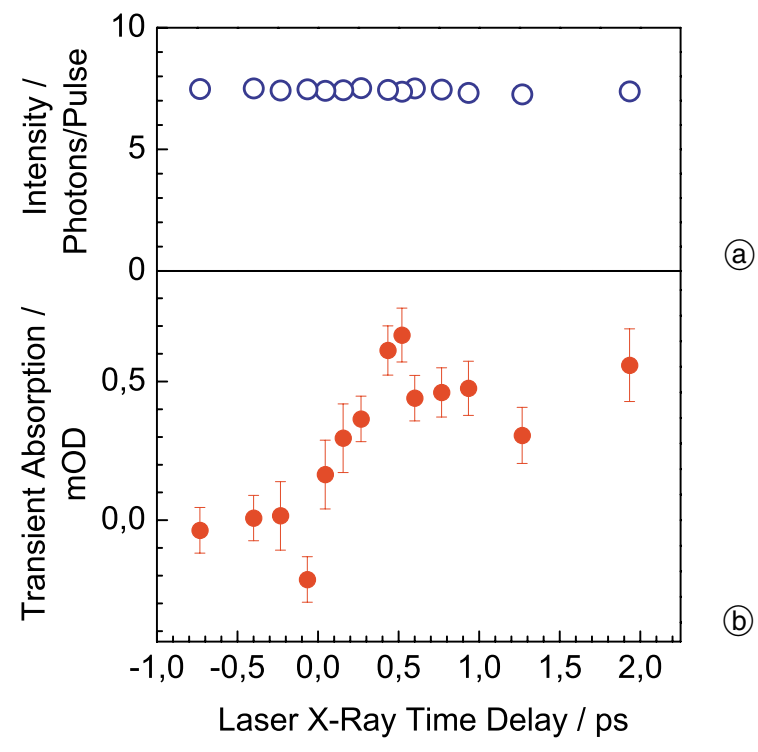

Fig. 20. Preliminary femtosecond transient XAFS of photoexcited $\left[\mathrm{Fe}^{\mathrm{II}}(\mathrm{bpy})_{3}\right]^{2+}$, (a) transient signal with $7.1 \mathrm{keV}$ probe x-radiation (at feature $B$ in Fig. 13a), (b) from this measurement derived single pulse intensity of the femtosecond X-ray pulses ( $=$ transmitted intensity, the incident intensity is ca. $20 \%$ higher). 
Fig. 20 was recorded over a collection time period of 7 hours).

This agreement is also extremely exciting, since we can now envision to measure trajectories of the molecular groups surrounding the central atom in photoexcited chromophores, and this breakthrough provides the grounds to estimate the feasibility of future femtosecond experiments of biological systems in their actual physiological media.

\subsection{Time-resolved XAFS in biology}

The above-mentioned capability of our XAFS setup to measure X-ray intensities with fluctuations only due to the statistical fluctuations of the incident pulse intensity puts us in a place to make more precise assessments about the overall feasibility of a time-resolved XAFS experiment, and to cross check these results with those obtained in different experiments (e.g., those reviewed here). Earlier, we have already performed this check for chemical solutes in the 0.1-2 $\mathrm{M}$ range [1]. We found that for transition metal compounds one requires about $I_{0}=10^{7}-10^{9}$ incident photons per data point, in order to measure transient changes of $\Delta \chi=1 \%$ relative to the absorption edge jump (Fig. 1) with a signal to noise ratio $S / N=1$, if one manages to excite $f=10 \%$ of the species. From these values we can then estimate the required number in a given real experiment by determining the actual fractional population $f$ and rescaling these numbers (e.g., by demanding a large value for $S / N$ ) [1].

For some of the XANES experiments shown earlier, we dealt with much larger changes than the aforementioned $\Delta \chi=1 \%$. This decreases the required number of photons considerably, into the $10^{5}-10^{6}$ range, which also depends on the excited state concentration obtainable in the experiment. For chemical species it is rather easy to increase the reactant concentration up several hundred $\mathrm{mM}$, while conventional amplified laser systems still have enough photons per pulse to generate a considerable excited state population.

When entering biological systems, e.g., proteins in physiological media, we can only work with concentrations up to the $\mathrm{mM}$ range. We have extended our calculations of Ref. [1] to include most elements in the periodic table, but now we no longer optimized the sample concentration, but kept it constant to $1 \mathrm{mM}$, and further fixed the sample thickness to $d=0.1 \mathrm{~mm}$. The result of this estimate is shown in Fig. 21. Hereby we solvate in water, as it is the physiological solvent. However, it can be changed and other solvents containing first-row elements deliver nearly identical values. Heavier elements in the protein are too dilute to considerably change these results. The graph shows two regions, the black shaded area for measuring at the atom's $K$ edge, and the red area for $L$ edge spectroscopy. The boundaries for each shell are for $\Delta \chi=1 \%$ (upper) and $\Delta \chi=10 \%$ (lower), which roughly correspond to EXAFS and XANES type of experiments, respectively. Both curves bend rather steeply upwards at lower $\mathrm{Z}$ because of the increasing solvent absorption at the respective edge energies, and bend slightly upwards for increasing $\mathrm{Z}$ due to the gradually decreasing $\mathrm{X}$-ray absorption cross section with increasing $\mathrm{Z}$.

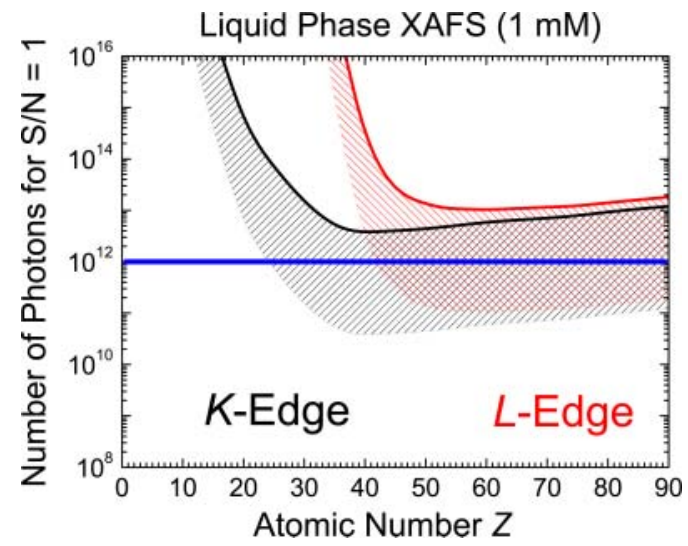

Fig. 21. Feasibility range for time-resolved XAFS of biologically relevant molecules in aqueous solutions, given in required incident photons per data point as a function of the X-ray absorbing element in the molecular system ( $1 \mathrm{mM}$ sample concentration and $0.1 \mathrm{~mm}$ sample thickness). $K$ edge spectroscopy can access lower-Z systems (black shaded region) than $L$ edge XAFS due to the solvent absorption properties (for details see text). Future XFELs will deliver intensities around $10^{12}$ photons/pulse (blue line).

For a desired experiment one picks the solid (upper) boundary curve in Fig. 21 corresponding to the selected absorption edge, reads off its calculated required incident number of photons (per data point) $I_{\text {calc }}$ and determine the required incident values $\left(I_{0}\right)$ via

$$
I_{0}=I_{\text {calc }} \cdot\left[\frac{S / N}{f / 0.1 \cdot \Delta \chi / 0.01}\right]^{2} \text {. }
$$

Overall, one will require up to many orders of magnitude more photons than for an experiment on chemical systems including transition metal or coordination chemistry compounds. While this will remain a challenge to observe with conventional $3^{\text {rd }}$ generation synchrotrons, future X-ray free electron lasers (XFELs) currently under construction will deliver single pulse intensities as demanded from Fig. 21.

\section{Conclusions}

This work shows examples of picosecond X-ray absorption spectroscopy applied to chemical systems in liquids. Time-resolved XAFS provides detailed information about the electronic state of short-lived reaction intermediates. Already knowledge of the oxidation state can aid in understanding the whereabouts of the photoexcited electron, as shown for the case of $\left[\mathrm{Ru}^{\mathrm{II}}(\mathrm{bpy})_{3}\right]^{2+}$, but also about possible fractional (re)population of valence states, as shown for the nascent iodine atoms in water. But XAFS methods are capable of simultaneously monitoring the geometric structure with high spatial resolution, as illustrated for the high-spin state of aqueous $\left[\mathrm{Fe}^{\mathrm{II}}(\mathrm{bpy})_{3}\right]^{2+}$.

These properties make this new tool suitable to study various phenomena in rather complex systems, even in disordered media. Thus it appears now feasible to study the very first instants of biological activity of proteins in physiological media. Ultrafast X-ray absorption spectroscopy holds a high potential to embark into structural dynamics in condensed phase systems. This is finally illustrated in a first femtosecond resolved X-ray absorption 
experiment. With the new powerful femtosecond X-ray sources becoming available these days, it is also possible to study nuclear wave packet dynamics exploiting XAFS tools.

Acknowledgments. We thank our collaborators, especially D. Grolimund, S. L. Johnson, P. Beaud and G. Ingold at the Swiss Light Source and their technical support staff, R. Facone and P. A. Heimann at the Advanced Light Source, A. Hauser (Uni Genève), F. M. F. de Groot (Uni Utrecht), M. Benfatto (INFN Rome), next to our former and current group members and collaborators listed in Refs. [17, 44]. These studies were funded by the Swiss National Science Foundation, via contracts 620-066145, 200021-107956, PP002-110464, 200020-116023, 200021-105239, and 200020116533 .

\section{References}

[1] Bressler, C.; Chergui, M.: Ultrafast X-ray Absorption Spectroscopy. Chem. Rev. 104 (2004) 1781-1812.

[2] Chen, L. X.; Jäger, W. J. H.; Jennings, G.; Gosztota, D. J.; Munkholm, A.; Hessler, J. P.: Capturing a photoexcited molecular structure through time-domain X-ray absorption fine structure. Science 292 (2001) 262-264.

[3] Chen, L. X.; Zhang, X.; Wasinger, E. C.; Attenkofer, K.; Jennings, G.; Muresan, A. Z.; Lindsey, J. S.: Tracking electrons and atoms in a photoexcited metalloporphyrin by X-ray transient absorption spectroscopy. J. Am. Chem. Soc. 129(31) (2007) 9616-9618.

[4] Khalil, M.; Marcus, M. A.; Smeigh, A. L.; McCusker, J. K.; Chong, H. H. W.; Schoenlein, R. W.: Picosecond X-ray absorption spectroscopy of a photoinduced iron(II) spin crossover reaction in solution. J. Phys. Chem. A110 (2006) 38-44.

[5] Gawelda, W.; Pham, V.-T.; Benfatto, M.; Zaushitsyn, Y.; Kaiser, M.; Grolimund, D.; Johnson, S. L.; Abela, R.; Hauser, A.; Bressler, C.; Chergui, M.: Structural determination of a shortlived excited iron(II) complex by picosecond X-ray absorption spectroscopy. Phys. Rev. Lett. 98 (2007) 057401.

[6] Koningsberger, D. C.; Prins, R. (Eds.): X-ray Absorption: Principles, Applications, Techniques of EXAFS, SEXAFS, and XANES. In: Chemical Analysis. Vol. 92, John Wiley \& Sons (1988).

[7] Rehr, J. J.; Albers, R. C.: Theoretical approaches to X-ray absorption fine structure. Rev. Mod. Phys. 72 (2000) 621.

[8] Saes, M.; Abela, R.; Grolimund, D.; Johnson, S. L.; Heimann, P. A.; Bressler, C.; Chergui, M.: Ultrafast Time-Resolved X-ray Absorption Spectroscopy of Chemical Systems. Synchr. Rad. News 16 (4) (2003) 12-20.

[9] Saes, M.; van Mourik, F.; Gawelda, W.; Kaiser, M.; Chergui, M.; Bressler, C.; Grolimund, D.; Abela, R.; Glover, T. E.; Heimann, P. A.; Schoenlein, R. W.; Johnson, S. L.; Lindenberg, A. M.; Falcone, R. W.: A Setup for Ultrafast Time-Resolved Xray Absorption Spectroscopy. Rev. Sci. Instrum. A75(1) (2004) $24-30$.

[10] Gawelda, W.; Pham, V.-T.; El Nahhas, A.; Kaiser, M.; Zaushitsyn, Y.; Johnson, S. L.; Grolimund, D.; Abela, R.; Hauser, A.; Bressler, C.; Chergui, M.: Capturing Transient Electronic and Molecular Structures in Liquids by Picosecond X-ray Absorption Spectroscopy. AIP Conf. Proc. 882 (2007) 31-36.

[11] Gawelda, W.; Bressler, C.; Saes, M.; Kaiser, M.; Tarnovsky, A. N.; Grolimund, D.; Johnson, S. L.; Abela, R.; Chergui, M.: Picosecond Time-Resolved X-ray Absorption Spectroscopy of Solvated Organometallic Complexes. Physica scripta $T \mathbf{1 1 5}$ (2005) 102-106.

[12] The utilized gated integrator has a memory effect of the previously triggered output value (due to a non-compete discharge of a capacitor) of ca. 5\%, which we also confirmed experimentally. In order to suppress this effect we provided the gated integrator with an additional trigger signal prior to each background measurement trigger to suppress this effect to below $0.3 \%$. Thus we triggered the gated integrator with a $6 \mathrm{kHz}$ trigger signal, while the ADC card was triggered for readout at $4 \mathrm{kHz}$, as described.
[13] Hagfeld, A.; Grätzel, M.: Light-induced redox reactions in nanocrystalline systems. Chem. Rev. 95 (1995) 49-68.

[14] Cannizzo, A.; Van Mourik, F.; Gawelda, W., Zgrablic, G.; Bressler, C.; Chergui, M.: Broadband femtosecond fluorescence spectroscopy of $\left[\mathrm{Ru}(\mathrm{bpy})_{3}\right]^{2+}$. Angew. Chem. Intl. Ed. 45 (2006) 3174.

[15] Saes, M.; Bressler, C.; Abela, R.; Grolimund, D.; Johnson, S. L.; Heimann, P. A.; Chergui, M.: Observing photochemical transients by ultrafast X-ray absorption spectroscopy. Phys. Rev. Lett. 90 (2003) 474031.

[16] Benfatto, M.; Della Longa, S.; Hatada, K.; Hayakawa, K.; Gawelda, W.; Bressler, C.; Chergui, M.: A full multiple scattering model for the analysis of time-resolved X-ray difference absorption spectra. J. Phys. Chem. B110 (2006) 14035.

[17] Tarnovsky, A. N.; Gawelda, W.; Johnson, M.; Bressler, C.; Chergui, M.: Photoexcitation of Aqueous Ruthenium(II)-tris-(2,2'-bipyridine) with High-Intensity Femtosecond laser Pulses. J. Phys. Chem. B110 (2006) 26497-26505.

[18] Gawelda, W.; Johnson, M.; de Groot, F. M. F.; Abela, R.; Bressler, C.; Chergui, M.: Electronic and molecular structure of photoexcited $\left[\mathrm{RuII}(\mathrm{bpy})_{3}\right]^{2+}$ probed by picosecond X-ray absorption spectroscopy. J. Am. Chem. Soc. 128 (2006) 5001.

[19] De Siervo, A.; Landers, R.; de Castro, S. G. C.; Kleiman, G. G.: Measurement of the $2 p$ XPS spectra of $4 \mathrm{~d}$ metals: $\mathrm{Nb}$ to $\mathrm{Sb}$. J. Electron Spectrosc. 88 (1998) 429-433.

[20] Ohno, M.; van Riessen, G. A.: Hole-lifetime width: a comparison between theory and experiment. J. Electron Spectrosc. 128 (2003) 1-31.

[21] Saes, M.: Picosecond X-ray absorption spectroscopy : application to coordination chemistry compounds in solution. $\mathrm{PhD}$ thesis No. 2970, EPF Lausanne, Switzerland, 2004.

[22] In a final step, we allowed all parameters (including widths) to vary freely, which neither improved the quality of the fit nor altered their values significantly.

[23] De Groot, F. M. F.: X-ray absorption and dichroism of transition metals and their compounds. J. Electron Spectrosc. 67 (1994) $529-622$.

[24] De Groot, F. M. F.: Multiplet effects in X-ray spectroscopy. Coord. Chem. Rev. 249 (2005) 31-63.

[25] De Groot, F. M. F.; Hocking, R. K.; Piamonteze, C.; Hedman, B.; Hodgson, K. O.; Solomon, E. I.: New developments in charge transfer multiplet calculations: Projection operators, mixed-spin states and $p$-bonding. AIP Conference Proceedings 882 (2007) 123-125.

[26] Bressler, C.; Saes, M.; Chergui, M.; Abela, R.; Pattison, P.: Optimizing a Time-Resolved X-ray Absorption Experiment. Nucl. Instrum. Meth. A467-468 (2001) 1444-1446.

[27] Bressler, C.; Chergui, M; Pattison P.; Wulff M.; Filipponi, A.; Abela, R.: Laser and Synchrotron radiation Experiment. Proc. SPIE 3451 (1998) 108-116.

[28] Bressler, C.; Saes, M.; Chergui, M.; Grolimund, D.; Abela, R.; Pattison, P.: Towards Structural Dynamics in Condensed Chemical Systems Exploiting Ultrafast Time-Resolved X-ray Absorption Spectroscopy. J. Chem. Phys. 116 (2002) 2955-2966.

[29] Alfano, J. C.; Walhout, P. K.; Kimura, Y.; Barbara, P. F.: Ultrafast transient-absorption spectroscopy of the aqueous solvated electron. J. Chem. Phys. 98 (1993) 5996-5998.

[30] Kloepfer, J. A.; Vilchiz, V. H.; Lenchenkov, V. A.; Germaine, A. C.; Bradforth, S. E.: The ejection distribution of solvated electrons generated by the one-photon photodetachment of aqueous I- and two-photon ionization of the solvent. J. Chem. Phys. 113 (2000) 6288-6307.

[31] Vilchiz, V. H.; Kloepfer, J. A.; Germaine, A. C.; Lenchenkov, V. A.; Bradforth, S. E.: Ultrafast thermalization dynamics of hot photoelectrons injected into water. Springer Series in Chemical Physics 66 (2001) 467-469.

[32] Pham, V.-T.; Gawelda, W.; Zaushitsyn, Y.; Kaiser, M.; Grolimund, D.; Johnson, S. L.; Abela, R.; Bressler, C.; Chergui, M.: Observation of the Solvent Shell Reorganization around Photoexcited Atomic Solutes by Picosecond X-ray Absorption Spectroscopy. J. Am. Chem. Soc. 129 (2007) 1530-1531.

[33] Tanida, H.; Kato, K.; Watanabe, I.: Hydrogen atom position in hydrated iodide anion from X-ray Absorption Near Edge Structure. Bull. Chem. Soc. Jpn 76 (2003) 1735. 
[34] Hertlein, M. P.; Adaniya, H.; Amini, J.; Bressler, C.; Feinberg, B.; Kaiser, M.; Neumann, N.; Prior, M. H.; Belkacem, A.: Inner-Shell Ionization of Potassium Atoms Ionized by a Femtosecond Laser. Phys. Rev. A73 (2006) 062715.

[35] Pham, V.-T.; et al.: To be published.

[36] Lawson Daku, L. M.; Vargas, A.; Hauser, A.; Fouqueau, A.; Casida, M. E.: Assessment of density functionals for the highspin/low-spin energy difference in the low-spin iron(II) tris $\left(2,2^{\prime}\right.$ bipyridine) complex. Chem. Phys. Chem. 6 (2005) 1393.

[37] Hauser, A.; Enachescu, C.; Daku, M. L.; Vargas, A.; Amstutz, N.: Low-temperature lifetimes of metastable high-spin states in spin-crossover compounds: The rule and exceptions to the rule. Coord. Chem. Rev. 250 (2006) 1642-1652.

[38] Brady, C.; McGarvey, J. J.; McCusker, J. K.; Toftlund, H.; Hendrickson, D. N.: Time-resolved relaxation studies of spin crossover systems in solution. Top. Curr. Chem. 235 (2004) 1.

[39] Gawelda, W.; Cannizzo, A.; Pham, V.-T.; Van Mourik, F.; Bressler, C.; Chergui, M.: Ultrafast Nonadiabatic Dynamics of $\left[\mathrm{FeII}(\mathrm{bpy})_{3}\right]^{2+}$ in Solution. J. Am. Chem. Soc. 129 (2007) 8199-8206.

[40] Hannay, C.; Hubin-Franskin, M. J.; Grandjean, F.; Briois, V.; Itie, J. P.; Polian, A.; Trofimenko, S.; Long, G. J.: X-ray absorption spectroscopic study of the temperature and pressure dependence of the electronic spin states in several iron(II) and cobalt(II) tris(pyrazolyl)borate complexes. Inorg. Chem. 36 (1997) 5580-5588.

[41] Briois, V.; Sainctavit, P.; Long, G. J.; Grandjean, F.: Importance of photoelectron multiple scattering in the iron K-edge X-ray absorption spectra of spin-crossover complexes: Full multiple scattering calculations for several iron(II) trispyrazolylborate and trispyrazolylmethane complexes. Inorg. Chem. 40 (2001) 912-918.

[42] Oyanagi, H.; Tayagaki, T.; Tanaka, K.: Photo-induced phase transitions probed by X-ray absorption spectroscopy: Fe(II) spin crossover complex. J. Phys. Chem. Solids 65 (2004) 14851489.

[43] Oyanagi, H.; Tayagaki, T.; Tanaka, K.: Synchrotron radiation study of photo-induced spin-crossover transitions: Microscopic origin of nonlinear phase transition. J. Luminescence 119 (2006) $361-369$.
[44] Gawelda, W.; Cannizzo, A.; Pham, V.-T.; El Nahhas, A.; Milne, C. J.; Van Der Veen, R.; Bressler, C.; Chergui, M.: Light-Induced Spin Crossover Probed by Ultrafast Optical and X-ray Spectroscopies. Chimia 61 (2007) 179-183.

[45] Ankudinov, A. L.; Ravel, B.; Rehr, J. J.; Conradson, S. D.: Pair distribution function and X-ray absorption signatures of rotational and radial local distortions in a model system with average long-range order. Phys. Rev. B58 (1998) 7565-7576.

[46] Ankudinov, A. L.; Bouldin, C. E.; Rehr, J. J.; Sims, J.; Hung, H.: Parallel calculation of electron multiple scattering using Lanczos algorithms. Phys. Rev. B65 (2002) 104107.

[47] Dick, S.: Crystal structure of tris(2,2'-bipyridine)iron(II) bis(hexafluorophosphate), $\left(\mathrm{C}_{10} \mathrm{H}_{8} \mathrm{~N}_{2}\right)_{3} \mathrm{Fe}\left(\mathrm{PF}_{6}\right)_{2}$. Z. Kristallogr. NCS 213 (1998) 356.

[48] Gawelda, W.: Time-resolved X-ray absorption specroscopy of transitionmetal complexes. Ph.D. thesis No. 3673, EPF Lausanne, Switzerland, 2006.

[49] Benfatto, M.; Congiu-Castellano, A.; Daniele, A.; Della Longa, S.: MXAN: A new software procedure to perform geometrical fitting of experimental XANES spectra. J. Synchr. Rad. 8 (2001) 267.

[50] Benfatto, M.; D’Angelo, P.; Della Longa, S.; Pavel, N. V.: Evidence of distorted fivefold coordination of the $\mathrm{Cu}^{2+}$ aqua ion from an X-ray-absorption spectroscopy quantitative analysis. Phys. Rev. B65 (2002) 174205.

[51] Tyson, T. A.; Hodgson, K. O.; Natoli, C. R.; Benfatto, M.: General multiple-scattering scheme for the computation and interpretation of X-ray-absorption fine structure in atomic clusters with applications to $\mathrm{SF}_{6}, \mathrm{GeCl}_{4}$, and $\mathrm{Br}_{2}$ molecules. Phys. Rev. B46 (1992) 5997.

[52] Stern, E. A.; Sayers, D. E.; Lytle, F. W.: Extended X-ray absorption fine structure technique. III. Determinatio of physical parameters. Phys. Rev. B11 (1975) 4836-4846.

[53] Gawelda, W.; et al.: To be published.

[54] Beaud, P.; Johnson, S. L.; Streun, A.; Abela, R.; Abramsohn, D.; Grolimund, D.; Krasniqi, F.; Schmidt, T.; Schlott, V.; Ingold, G.: Spatiotemporal Stability of a Femtosecond Hard-X-Ray Undulator Source Studied by Control of Coherent Opticol Phonons. Phys. Rev. Lett 99 (17) (2007) 174801/1.

[55] Bressler, C.: To be published. 